\title{
Zero-temperature phase diagram of Bose-Fermi gaseous mixtures in optical lattices
}

\author{
T. P. Polak \\ Faculty of Physics, Adam Mickiewicz University of Poznań, Umultowska 85, 61-614 Poznań, Poland \\ T. K. Kopeć \\ Institute for Low Temperatures and Structure Research, \\ Polish Academy of Sciences, P.O. Box 1410, 50-950 Wroclaw 2, Poland
}

\begin{abstract}
We study the ground state phase diagram of a mixture of bosonic and fermionic cold atoms confined on two- and three-dimensional optical lattices. The coupling between bosonic fluctuations and fermionic atoms can be attractive or repulsive and has similarities with electron-phonon coupling in crystals. We investigate behavior of the mixtures in the limit, where the Bogoliubov sound velocity that dictates bosonic dynamics is comparable to the Fermi velocity, hence the retardation effects are important part of the physics. The dynamic Lindhard response function of the fermionic density to changes in the bosonic number of particles above some critical frequency can alter the sign and in consequence the inter-species interaction between particles becomes repulsive in contrast to the static limit (instantaneous and always attractive). Considering the above we show that the structure of the phase diagrams crucially depends on the difference in masses of the bosons and fermions. We discuss the situations where integrating out fermionic field provides an additional interaction that can decrease or increase bosonic coherence.
\end{abstract}

PACS numbers: 03.75.Lm, 05.30.Jp, 03.75.Nt

\section{INTRODUCTION}

Trapping and cooling Bose-Fermi mixtures of dilute quantum gases has opened a wide area of research in atomic physics. The interactions between bosonic and fermionic species interconnect two systems of fundamentally different quantum statistics. The diluteness of the gaseous mixtures allows one to treat the interactions between particles in terms of binary collisions. In consequence we can replace the real inter-atomic potential by a pseudo-potential characterized by only one parameter, the $s$-scattering length. The latter is experimentally 1 4 tunable by exploiting optically or magnetically induced Feshbach resonances 5 . Despite its simplicity the interaction potential (mathematically ill defined [6]) of the ultra-cold multi-component gases confined in optical lattices is responsible for a wealth of novel quantum phases [7, 8, including charge density waves $(\mathrm{CDW})[9,10$, as well as supersolid behavior [11-13]. The nature of the phase transition and qualitative phase diagram for one-component bosonic system can be inferred based on very simple arguments[14. When tunneling between lattice sites of the bosons is suppressed, compared with point-like interaction between them, the system can undergo a quantum phase transition between a superfluid (SF) phase (characterized by large number fluctuations at each lattice site), and a Mott insulating (MI) phase where each lattice site is occupied by precisely an integer number of bosons without any number fluctuations. Adding to a bosonic system a fermionic ingredient and allowing for the mutual repulsion or attraction between

\footnotetext{
*Electronic address: tppolak@amu.edu.pl
}

species of different statistics strongly affects the equilibrium properties. Increasing the boson-fermion repulsion drives the system towards spatial separation whereas attraction gives rise to implosion [15, 16. The dynamics underlying the phase transitions in the Bose-Fermi mixtures is produced by the small changes of the bosonic density which induce a modulation of the fermionic density. As a consequence of the feedback of the fermionic perturbation a shift of the bosonic energy occurs, thereby inducing an additional attraction or repulsion that changes the original interaction between bosons [17. In the case of deep optical lattices and small densities, the coherent description of the system provided by the Gross-Pitaevskii equation is not reliable due to rising effects of correlation. The experimental data clearly demonstrated that adding a fermionic cloud to strongly interacting bosons always results in a decay of visibility of the interference pattern in time-of-flight images 1, 3, 4. Moreover, the scale of disappearance of the coherence in the mixtures is different for attractive and repulsive scattering lengths 4 . To predict such behavior theoretically one can include the more general, than one-component Bose-Hubbard $(\mathrm{BH})$, multi-band model. In mentioned approach if the higher-band renormalization of the boson parameters is dominant over the fermion screening of the interaction, the Mott-insulating lobes in the Bose-Hubbard phase diagram are enhanced for either sign of the Bose-Fermi interactions 18, 19. On the other hand inclusions of the retardation effects 20] (which arises from the presence of very low energy excitations in a Fermi sea) give rise to so-called orthogonality catastrophe 21. Another approach to quantum mixtures of particles of unequal masses, when the difference in the tunneling amplitudes between heavy bosons and light fermions is large enough to neglect quantum nature of the bosons, provides to 
description of the system in Fermi-Bose version of the Falicov-Kimball model[22]. The mutual interactions of bosons and fermions can affect the spectrum of collective excitations in the collisionless regime as the mixture goes toward either demixing or collapse [15]. It has been shown that mode-mode coupling effects may arise when sound velocity of the Bose gas is comparable to the Fermi velocity of the fermions 23. The energy spectra of the bosonic and fermionic mixtures and phase diagram were also obtained by the field theory methods 24, however calculated phase boundary does not change the structure and only shifts the chemical potential. The properly constructed effective theory lead to an effective, fermion mediated, long-range interaction between bosons with alternating sign that is the origin of the CDW and can explain the MI-CDW phase separation 9. There is also another possibility of the analysis of the mixtures of atoms with different statistics where the second species is strongly localized on random sites which can lead to random shifts of the on-site energies and, in consequence, the disorder with discrete probability distribution is created 25]. Recently the experiments [26] on a harmonically trapped mixtures of atomic bose-bose gases show that the presence of relevant fraction of the ${ }^{41} \mathrm{~K}$ bosonic species modifies the quantum phase transition occurring in ${ }^{87} \mathrm{Rb}$ inducing a significant loss of coherence similarly to bose-fermi systems that can be explained in the meanfield theory framework[27].

The aim of this work is to study the superfluid to Mottinsulator zero-temperature phase transition by means of the Bose-Fermi-Hubbard model in two- $(2 D)$ and threedimensional $(3 D)$ optical condensates. In order to find a phase boundary for BF mixtures very sophisticated methods and calculations are required. Only several theoretical works concentrated on the inherent difficulty of dealing with BF Hubbard Hamiltonian originates from the non-perturbative nature of the model and retardation effects. To elucidate the quantum phase transition in optical lattices, where the kinetic energy scale is less than the dominating repulsive energy and density-density coupling between species with different statistics comes into play, we have adopted a theoretical approach for strongly interacting fermions 28] to the BF Hubbard model in a way to include the effects of particle number fluctuations and make the qualitative phase diagrams more quantitative [29]. To facilitate this task, we employ a functional integral formulation of the theory that enables to perform functional integration over fields defined on different topologically equivalent classes of the $\mathrm{U}(1)$ group, i.e., with different winding numbers. An inclusion of the winding numbers (comes from periodicity of the phase variables) is unavoidable in order to properly construct the phase diagram and the Poisson re-summation formula turns out to be very useful for derivation of the topological term of the partition function. The quantum rotor representation method we use is deeply rooted in the gauge symmetries of the model. We construct an invariant theory introducing an appropriate $\mathrm{U}(1)$ gauge transformation. In Sec. II we review the Hamiltonian for the system and show the connections of the parameters to the experimentally measured quantities in optical lattices. Sec. III contains description of the method we use and can serve a guidance to obtain the critical line equation presented in Sec. IV. Before showing the phase diagrams for the Bose-Fermi Hubbard model in the quantum rotor description we make some general remarks in Sec. V concern the phase boundary equation and compare our results with the diagrammatic perturbation approach to the one-component Bose-Hubbard model for experimentally accessible densities of the particles. Sec. VI presents the discussion of the ground state phase diagrams for the Bose-Fermi Hubbard Hamiltonian calculated within quantum rotor approach. The Sec. VII. is devoted to some concluding remarks. The appendixes contain the derivation of relevant formulas of the main text and are introduced to keep the text self-contained.

\section{HAMILTONIAN}

For bosons confined in optical lattices the two main energy scales are set by the hopping amplitude proportional to $t_{b}$ (which sets the kinetic energy scale for bosons) due to the particles tunneling, and the on-site interaction $U_{b}>0$. For $t_{b}>U_{b}$ the phases of the superfluid order parameter on individual lattice sites are well defined. On the other hand, for sufficiently large repulsive energy $U_{b}$, the quantum phase fluctuations lead to complete suppression of the long-range phase coherence even at zero temperature. The competition between the kinetic energy, which is gained by delocalizing bosons over lattice sites and the repulsive interaction energy, which disfavors having more than one particle at any given site, can be modeled by quantum Bose-Hubbard Hamiltonian[14]. The physics of the bosonic and non-interacting spin-polarized (collisions in the $s$-wave channel are forbidden by their statistics) fermionic mixtures with density-density interaction between species $U_{b f}$ leads to Bose-Fermi-Hubbard Hamiltonian [8]:

$$
\begin{aligned}
\mathcal{H} & =\frac{U_{b}}{2} \sum_{i} n_{b i}^{2}-\sum_{\langle i, j\rangle} t_{b i j} b_{i}^{\dagger} b_{j}-\bar{\mu}_{b} \sum_{i} n_{b i} \\
& -\sum_{\langle i, j\rangle} t_{f i j} f_{i}^{\dagger} f_{j}-\mu_{f} \sum_{i} n_{f i}+U_{b f} \sum_{i} n_{b i} n_{f i}
\end{aligned}
$$

where $b_{i}^{\dagger}\left(f_{i}^{\dagger}\right)$ and $b_{i}\left(f_{i}\right)$ stand for the bosonic (fermionic) creation and annihilation operators $n_{b i}=$ $b_{i}^{\dagger} b_{i},\left(n_{f i}=f_{i}^{\dagger} f_{i}\right)$ is the boson (fermion) number operator on the site $i$, and the reduced chemical potential $\bar{\mu}_{b}=\mu_{b}+U_{b} / 2$ controls the number of bosons and $\mu_{f}$ fermions respectively. Here, $\langle i, j\rangle$ identifies summation over the nearest-neighbor sites. Furthermore, $t_{b i j}\left(t_{f i j}\right)$ is the hopping matrix element for bosons (fermions). For simplicity, we neglect the inhomogeneous magnetic trap 
potential. If the on-site boson-fermion coupling strength $U_{b f}$ becomes very strong the dilute gaseous mixtures are unstable to phase separation $\left(U_{b f}>0\right)$ or to collapse of the phase separated configuration $\left(U_{b f}<0\right)$ [3, 30]. The presence of the lattice will introduce kinetic energy scales $t_{b(f)}$ competing with $U_{b f}$ stabilizing the system. We assume that an optical lattice created by the counterpropagating laser beams is deep enough and we can restrict ourselves to the lowest Bloch bands. The corresponding experimental parameters can be estimated by following relations 12

$$
\begin{aligned}
t_{x} & \simeq \frac{4}{\sqrt{\pi}} E_{r}^{x}\left(\frac{V_{0}}{E_{r}^{x}}\right)^{3 / 4} \exp \left[-2\left(\frac{V_{0}}{E_{r}^{x}}\right)\right], \\
U_{x} & \simeq \sqrt{\frac{8}{\pi}} k a_{x} E_{r}^{x}\left(\frac{V_{0}}{E_{r}^{x}}\right)^{3 / 4},
\end{aligned}
$$

(subscript $x=\{b, f\}$ means $b$ bosons and $f$ fermions respectively) where boson-boson $a_{b}$, fermion-fermion $a_{f}$ and boson-fermion $a_{b f}$

$$
U_{b f} \simeq \sqrt{\frac{8}{\pi}} k a_{b f} E_{r}^{b}\left(\frac{V_{0}}{E_{r}^{b}}\right)^{3 / 4} \frac{1+\frac{m_{b}}{m_{f}}}{\left(1+\sqrt{\frac{m_{b}}{m_{f}}}\right)^{3 / 2}}
$$

scattering lengths can be continuously tune in the experiments 1-4 inducing attractive or repulsive interaction between species. The $k=2 \pi / \lambda$ is the wavelength of the laser and $E_{r}^{x}=\hbar^{2} k^{2} / 2 m_{x}$ is the recoil energy and $m_{x}$ is the atomic mass.

\section{DESCRIPTION OF THE METHOD}

We write the partition function of the system switching from the particle-number representation to the conjugate phase representation of the bosonic degrees of freedom using the bosonic and fermionic path-integral over the complex fields $a_{i}(\tau)$ and $f_{i}(\tau)$ depending on the "imaginary time" $0 \leq \tau \leq \beta \equiv 1 / k_{\mathrm{B}} T$ with $T$ being the temperature:

$$
\mathcal{Z}=\int[\mathcal{D} \bar{b} \mathcal{D} b \mathcal{D} \bar{f} \mathcal{D} f] e^{-\mathcal{S}[\bar{b}, b, \bar{f}, f]}
$$

The action $\mathcal{S}$ is given by

$$
\mathcal{S}=\mathcal{S}_{B}[\bar{b}, b, \bar{f}, f]+\int_{0}^{\beta} d \tau \mathcal{H}(\tau)
$$

where

$$
\begin{aligned}
\mathcal{S}_{B}[\bar{b}, b, \bar{f}, f] & =\sum_{i} \int_{0}^{\beta} d \tau \bar{b}_{i}(\tau) \frac{\partial}{\partial \tau} b_{i}(\tau) \\
& +\sum_{i} \int_{0}^{\beta} d \tau \bar{f}_{i}(\tau) \frac{\partial}{\partial \tau} f_{i}(\tau) .
\end{aligned}
$$

In the next section we will integrate over the fermionic fields since the action is quadratic in $f_{i}(\tau)$ variables. We attempt to reduce the large number of degrees of freedom in the partition function to the few which dominate the low energy physics.

\section{A. Integration over fermionic fields}

Before integrating out of the fermionic degrees of freedom we write the action in the form:

$$
\begin{aligned}
\mathcal{S}_{b}\left[\bar{b}, b, n_{b}\right] & =\int_{0}^{\beta} d \tau\left\{\sum_{i}\left[\bar{b}_{i}(\tau) \frac{\partial}{\partial \tau} b_{i}(\tau)+\frac{U_{b}}{2} n_{b i}^{2}(\tau)\right]\right. \\
& \left.-\sum_{\langle i, j\rangle} t_{b i j} \bar{b}_{i}(\tau) b_{j}(\tau)-\bar{\mu}_{b} \sum_{i} n_{b i}(\tau)\right\}, \\
\mathcal{S}_{f}\left[\bar{f}, f, n_{f}\right] & =\int_{0}^{\beta} d \tau\left[\sum_{i} \bar{f}_{i}(\tau) \frac{\partial}{\partial \tau} f_{i}(\tau)\right. \\
& \left.+\sum_{\langle i, j\rangle} t_{f i j} \bar{f}_{i}(\tau) f_{j}(\tau)-\mu_{f} \sum_{i} n_{f i}(\tau)\right], \\
\mathcal{S}_{\text {int }}\left[n_{b}, n_{f}\right] & =U_{b f} \sum_{i} \int_{0}^{\beta} d \tau n_{b i}(\tau) n_{f i}(\tau) .
\end{aligned}
$$

We notice that adding the inter-species interaction term to the fermionic part of the action

$$
\mathcal{S}_{f+\mathrm{int}}=\sum_{i, j} \int_{0}^{\beta} d \tau d \tau^{\prime} \bar{f}_{i}(\tau)\left[\hat{G}_{f+\mathrm{int}}\left(\tau, \tau^{\prime}\right)\right]_{i j} f_{j}\left(\tau^{\prime}\right)
$$

allows one to integrate over fermionic fields, because the action is Gaussian in $f_{i}(\tau)$ operators. Resulting partition function takes the form

$$
\mathcal{Z}=\int[\mathcal{D} \bar{b} \mathcal{D} b] e^{-\mathcal{S}_{b}\left[\bar{b}, b, n_{b}\right]} e^{\operatorname{Tr} \ln \hat{G}_{f+\mathrm{int}}^{-1}}
$$

We will be looking for solutions obeying translational invariance in the "imaginary time" direction, i.e., such that the partition function depends only on the difference $\left|\tau-\tau^{\prime}\right|$. Expanding the trace of the logarithm in Eq. 10 we have

$$
\begin{aligned}
\operatorname{Tr} \ln \hat{G}_{f+\mathrm{int}}^{-1} & =-\operatorname{Tr} \ln \hat{G}_{f}-\operatorname{Tr} \hat{G}_{\mathrm{int}} \hat{G}_{f} \\
& -\frac{1}{2} \operatorname{Tr}\left(\hat{G}_{\mathrm{int}} \hat{G}_{f}\right)^{2}
\end{aligned}
$$

with

$$
\begin{aligned}
& {\left[\hat{G}_{f}^{-1}\left(\tau, \tau^{\prime}\right)\right]_{i j}=\left[\left(\frac{\partial}{\partial \tau}-\mu_{f}\right) \delta_{i j}-t_{f i j} I_{i j}\right] \delta\left(\tau-\tau^{\prime}\right),} \\
& {\left[\hat{G}_{\text {int }}\left(\tau, \tau^{\prime}\right)\right]_{i j}=U_{b f} \bar{b}_{i}(\tau) b_{j}\left(\tau^{\prime}\right) \delta_{i j} \delta\left(\tau-\tau^{\prime}\right) .}
\end{aligned}
$$


We defined $I_{i j}=1$ if $i, j$ are the nearest neighbors and equals zero otherwise. Trace over first term of the expansion gives constant contribution of the fermions, in the non-interacting system, to the action. Second one induces a shift in the chemical potential of bosons. The third term after exploiting Fourier-Matsubara transform

$$
b_{i}(\tau)=\frac{1}{N \beta} \sum_{\boldsymbol{k}, \ell} b_{\boldsymbol{k}}\left(\omega_{\ell}\right) e^{-i\left(\omega_{\ell} \tau-\boldsymbol{k} \cdot \boldsymbol{r}_{i}\right)},
$$

where $\omega_{\ell}=2 \pi \ell / \beta\left(\nu_{\ell}=\pi(2 \ell+1) / \beta\right)$ with $(\ell=$ $0, \pm 1, \pm 2, \ldots)$ are Bose(Fermi)-Matsubara frequencies respecting periodic (anti-periodic) boundary conditions of the bosonic (fermionic) field operator, reduces to

$$
\begin{aligned}
\operatorname{Tr} \ln \hat{G}_{f+\mathrm{int}}^{-1} & =\frac{U_{b f}^{2}}{2} \sum_{\boldsymbol{q}, \ell} \Lambda_{\boldsymbol{q}}\left(\omega_{\ell}\right) \\
& \times \chi\left(\boldsymbol{q}, i \nu_{\ell}, \mu_{f}, \beta\right) \Lambda_{-\boldsymbol{q}}\left(-\omega_{\ell}\right),
\end{aligned}
$$

where $\Lambda_{\boldsymbol{q}}\left(\omega_{\ell}\right)=\bar{b}_{\boldsymbol{q}}\left(\omega_{\ell}\right) b_{\boldsymbol{q}}\left(\omega_{\ell}\right)$ and $\chi\left(\boldsymbol{q}, i \nu_{\ell}, \mu_{f}, \beta\right)$ is called Lindhard function

$$
\chi\left(\boldsymbol{q}, i \nu_{\ell}, \mu_{f}, \beta\right)=\sum_{\mathbf{k}} \frac{f\left[t_{f \boldsymbol{k}}, \mu_{f}, \beta\right]-f\left[t_{f \boldsymbol{k}+\boldsymbol{q}}, \mu_{f}, \beta\right]}{t_{f \boldsymbol{k}}-t_{f \boldsymbol{k}+\boldsymbol{q}}-i \nu_{\ell}} .
$$

In the above $f\left[t_{f \boldsymbol{k}}, \mu_{f}, \beta\right]=1 /\left\{\exp \left[\beta\left(t_{f \boldsymbol{k}}-\mu_{f}\right)\right]+1\right\}$ is the Fermi distribution function and $t_{f \boldsymbol{k}}$ is the fermionic dispersion relation. To stay in the local (momentum integrated) regime we perform $\boldsymbol{q}$ and $\mathbf{k}$ integration over the first Brillouin zone and in the $T \rightarrow 0$ limit using an analytic continuation $i \nu_{\ell} \rightarrow \omega+i \epsilon$ we obtain imaginary part $\chi^{\prime \prime}\left(\omega, \mu_{f}\right) \equiv \operatorname{Im} \chi\left(\omega, \mu_{f}\right)$ of the local dynamic Lindhard function (see Appendix for details)

$$
\begin{aligned}
\chi^{\prime \prime}\left(\omega^{\prime}, \mu_{f}\right) & =\int_{-\infty}^{+\infty} d x\left[\Theta\left(x-\omega^{\prime}-\mu_{f}\right)-\Theta\left(x-\mu_{f}\right)\right] \\
& \times \rho(x) \rho\left(x-\omega^{\prime}\right)
\end{aligned}
$$

that satisfies sum rule which is just the conservation of the number of particles. In the above $\rho(\xi)=$ $N^{-1} \sum_{\mathbf{k}} \delta\left[\xi-t_{f \boldsymbol{k}}\right]$ is the density of states and $\Theta(x)$ is the unit step function. Therefore, the corresponding real part $\chi^{\prime}\left(\omega, \mu_{f}\right) \equiv \operatorname{Re} \chi\left(\omega, \mu_{f}\right)$ can be easily deduced from Kramers-Krönig relation

$$
\chi^{\prime}\left(\omega, \mu_{f}\right)=\frac{2}{\pi} \int_{0}^{+\infty} \frac{\omega^{\prime} \chi^{\prime \prime}\left(\omega^{\prime}, \mu_{f}\right)}{\omega^{\prime 2}-\omega^{2}} d \omega^{\prime} .
$$

Later, without any loss of generality, we drop the chemical potential dependence writing $\chi^{\prime}\left(\omega, \mu_{f}=0\right) \equiv \chi^{\prime}(\omega)$ and $\chi^{\prime \prime}\left(\omega, \mu_{f}=0\right) \equiv \chi^{\prime \prime}(\omega)$. Finally the "imaginarytime" partition function with integrated out fermionic degrees of freedom, in the local approximation (see Appendix for details), can be written as:

$$
\mathcal{Z}=\int[\mathcal{D} \bar{b} \mathcal{D} b] e^{-\mathcal{S}_{\text {eff }}[\bar{b}, b]}
$$
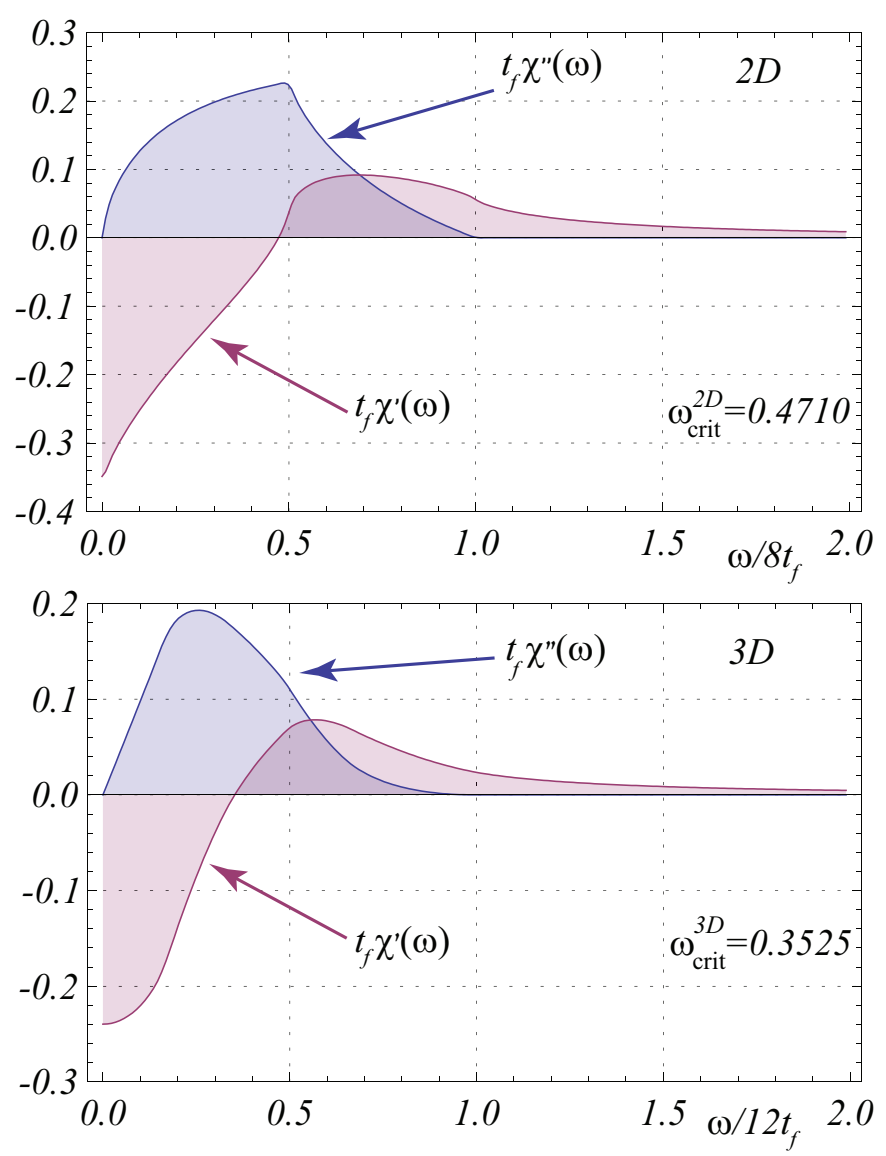

Figure 1: (Color online) Real $t_{f} \chi^{\prime}(\omega)$ and imaginary $t_{f} \chi^{\prime \prime}(\omega)$ part of the local dynamic Lindhard function for square $(2 D)$ and cubic lattice $(3 D)$, in the low-temperature limit, as a function of frequency, where the normalized fermionic potential $\mu_{f} / t_{f}=0$ is equal zero. The normalized value of the critical frequency $\omega_{\text {crit }}^{2 D, 3 D}$ shows where induced, frequencydependent, effective part of the interaction $U_{b f}^{2} \chi^{\prime}\left(\omega_{\text {crit }}\right)$ changes character from attractive to repulsive.

with the effective action

$$
\begin{aligned}
\mathcal{S}_{\text {eff }}[\bar{b}, b] & =\int_{0}^{\beta} d \tau\left\{\sum_{i} \bar{b}_{i}(\tau) \frac{\partial}{\partial \tau} b_{i}(\tau)\right. \\
& +\frac{U_{\text {eff }}}{2} \sum_{i} n_{b i}^{2}(\tau)-\sum_{\langle i, j\rangle} t_{b i j} \bar{b}_{i}(\tau) b_{j}(\tau) \\
& \left.-\bar{\mu}_{b} \sum_{i} n_{b i}(\tau)\right\}
\end{aligned}
$$

expressed in terms of bosonic degrees of freedom only. We want to emphasize that applying a local, in the Matsubara-imaginary time, approach we neglect any dissipation effects. Whereas locality in real space rules out some parts of interesting physics such as the charge density wave, namely an insulating phase with modulated density or the supersolid phase, presenting the coexistence of superfluidity and a periodic spatial modulation of the density, different from that of the lattice. On 
the other hand, the long-range character of the fermion mediated interaction between bosons with the fermioninduced mean field potential can lead to spatially homogeneous regions of commensurate CDW [9]. The motivation of the local approximation was the idea that response of an interacting system can be pictured as the response of a non-interacting system to an effective selfconsistent field, that depends on global properties such as the particle densities. A question of both fundamental and practical interest is, to what extent can the physics of the exact non-local interaction be captured by an approximate local theory? It seems that local approximations often work surprisingly well, yielding energies very accurately, without suffering from some of the characteristic drawbacks of non-locality 31.

From Eq. 19 it is concluded that there is a striking resemblance to the one-component Bose-Hubbard action with the original repulsive interaction replaced now by

$$
U_{b} \rightarrow U_{\text {eff }}=U_{b}+U_{b f}^{2} \chi^{\prime}\left(\omega, \mu_{f}\right)
$$

which is the induced, frequency-dependent, effective interaction between bosons. From Eq. 20 we see that integrating out fermionic field from BF Hubbard Hamiltonian provides an additional interaction among bosons, which is not affected by the attractive or repulsive nature of the inter-species interaction $\pm U_{b f}$. Before we proceed with further calculations let us make some remarks. The substitution we introduced in Eq. (20) is deceptively simple and can lead to the assumption that the phase diagram of the BFH model can be easily derived from the critical line of the one-component BH Hamiltonian, which already has been obtained in several approximations. Unfortunately, as we will see in the next section, $U_{b f}^{2} \chi^{\prime}\left(\omega, \mu_{f}\right)$ is not the only one ingredient to the final equation for the critical line. The additional part, which influences the phase boundary line condition, comes from the inter-species interaction and number of fermions added to the system has an impact on amplitude of the order parameter. Moreover, the chemical potential for fermions $\mu_{f} / t_{f}$ is shifted (we postpone calculations of it now and show proper formula later) because of the induced effective interaction between them.

Next step in the calculations depends on the ratio $m_{b} / m_{f}$ of the masses of bosons and fermions which can be seen from Eq. (4). Consequence of the latter is the fact that the speed of the Bogoliubov sound $c_{b}$ for bosons differs from the first sound $v_{f}$ of the ideal Fermi gas. In typical experimental realizations ${ }^{40} \mathrm{~K}-{ }^{87} \mathrm{Rb}$ systems the acoustic long-wavelength (boson) $c_{b}$ and fermion $v_{f}$ velocities are comparable or $c_{b} / v_{f}<1$ (boson mass is larger than fermion). Therefore we do not restrict our calculations to the static limit but consider also local dynamical response function (thus including the retardation effects). If $c_{b}$ is much larger than $v_{f}$ (in the ${ }^{40} \mathrm{~K}$ ${ }^{23} \mathrm{Na}$ species we have $c_{b} / v_{f} \sim 5$ ) the resulting interaction between bosons is instantaneous and always attractive (with $\left.U_{b f}^{2} \chi^{\prime}\left(\omega, \mu_{f}\right)<0\right)$ so using the static approximation is justified with an error which involves the small

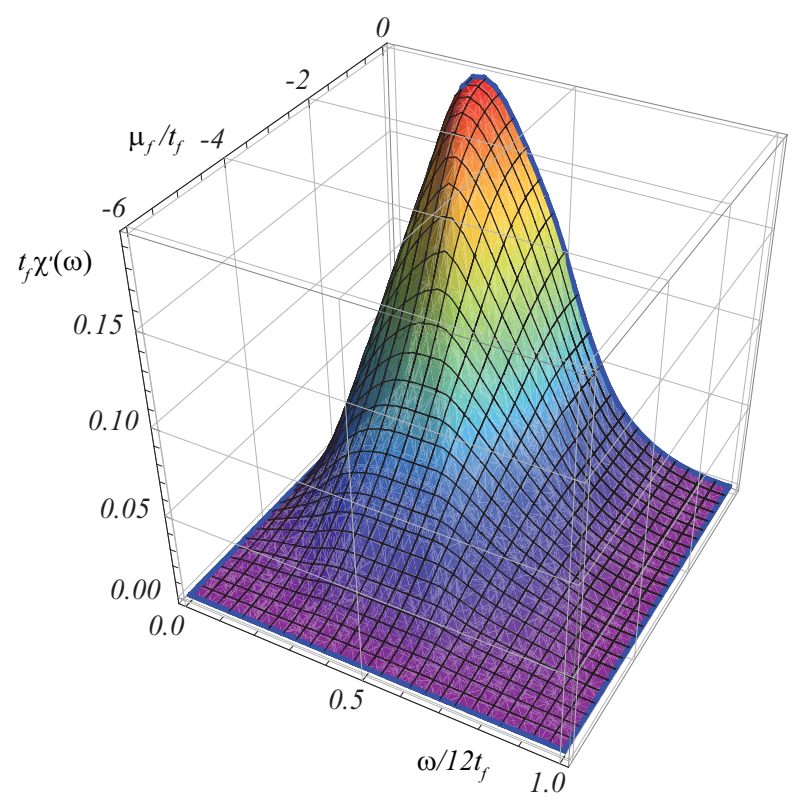

Figure 2: (Color online) Imaginary $t_{f} \chi^{\prime \prime}\left(\omega, \mu_{f}\right)$ part of the local dynamic Lindhard function for cubic lattice in the space of the parameters: normalized frequency $\omega / t_{f}$ and fermionic chemical potential $\mu_{f} / t_{f}$ in the zero-temperature limit.

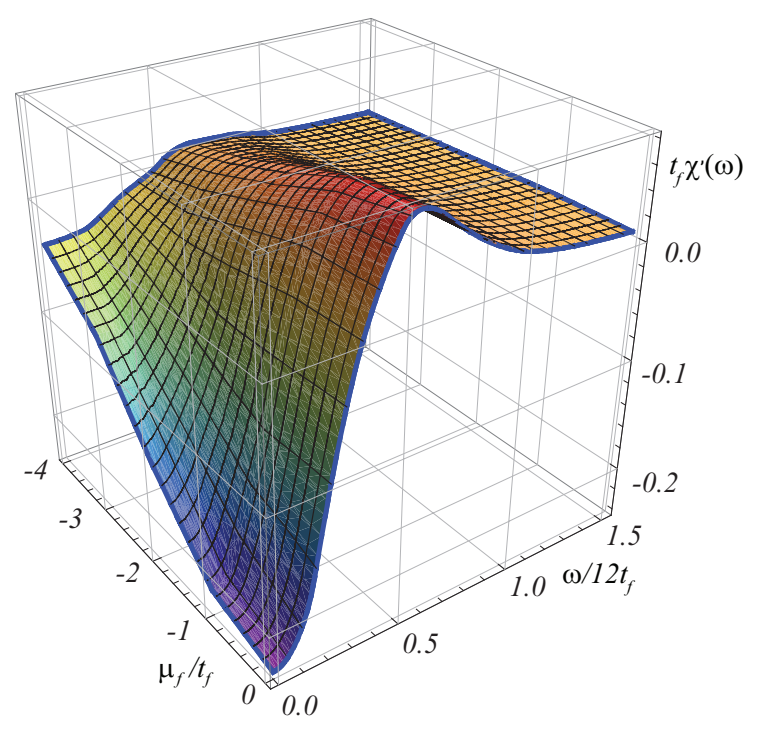

Figure 3: (Color online) Real $t_{f} \chi^{\prime}\left(\omega, \mu_{f}\right)$ part of the local dynamic Lindhard function for cubic lattice in the space of the parameters: normalized frequency $\omega / t_{f}$ and fermionic chemical potential $\mu_{f} / t_{f}$ in the zero-temperature limit.

parameter $v_{f} / c_{b}$.

The imaginary part of the Lindhard response function rises to a broad peak before falling and the real part takes zero when changes in $\chi^{\prime \prime}(\omega)$ are the biggest (see Fig. 1). The real part $\chi^{\prime}(\omega)$ is negative for the frequencies $\omega / 8 t_{f}<0.4710=\omega_{\text {crit }}^{2 D}$ for square and $\omega / 12 t_{f}<0.3525=\omega_{\text {crit }}^{3 D}$ for cubic lattice (see Fig. 11). We normalized the frequency by the width of the band 
for non-interacting fermions to show the scale of the energy. Nevertheless, for higher values of the normalized frequency $\omega>\omega_{\text {crit }}$ the induced part of the interaction $U_{b f}^{2} \chi^{\prime}(\omega)$ can be positive and increase the effective interaction between bosons, in consequence provide stronger localization these species on lattice sites. In the case of large fermion hopping and commensurate filling with the lattice the effective long-range density-density interaction between bosons has alternating sign and is the origin of the charge density-wave phases [9]. The higher values of the normalized chemical potential for the fermions $\mu_{f} / t_{f}$ decreases the values of both (Fig. 2 and Fig. 3 real and imaginary part of the local Lindhard function. That leads to the situation where the terms containing explicitly the average density of fermions $n_{\mathrm{F}}$ will acquire more significance than terms with exclusively the inter-species interaction $U_{b f}$.

\section{B. Static and periodic bosonic fields and gauge transformation}

Unfortunately the effective action is not quadratic in bosonic fields $b_{i}$ and we have to decouple the effective interaction term in Eq. 19p by a Gaussian integration over the auxiliary scalar potential fields

$$
V_{i}(\tau)=V_{i 0}+V_{i}^{\prime}(\tau),
$$

with static

$$
V_{i 0}=\frac{1}{\beta} V_{i}\left(\omega_{\ell=0}\right)
$$

and periodic part

$$
V_{i}^{\prime}(\tau)=\frac{1}{\beta} \sum_{\ell=1}^{+\infty} V_{i}\left(\omega_{\ell}\right) e^{i \omega_{\ell} \tau}+\text { c.c, }
$$

where $\omega_{\ell}$ is the Bose-Matsubara frequency. We observe now that effective BF Hubbard Hamiltonian has a local $\mathrm{U}(1)$ gauge symmetry, when expressed in terms of the underlying boson variables. This points out a possibility of an emergent dynamical U (1) gauge field as a fluctuating complex field attached to bosonic variables, which is dynamically generated, by interacting bosons. Thus, the periodic part $V_{i}^{\prime}(\tau) \equiv V_{i}^{\prime}(\tau+\beta)$ couples to the local particle number through the Josephson-like relation $\dot{\phi}_{i}(\tau)=V_{i}^{\prime}(\tau)$, where

$$
\dot{\phi}_{i}(\tau) \equiv \frac{\partial \phi_{i}(\tau)}{\partial \tau}=e^{-\phi_{i}(\tau)} \frac{1}{i} \frac{\partial}{\partial \tau} e^{\phi_{i}(\tau)} .
$$

The quantity $\phi(\tau)$ is the $\mathrm{U}(1)$ phase field and satisfies the periodicity condition $\phi_{i}(\beta)=\phi_{i}(0)$ as a consequence of the periodic properties of the $V_{i}^{\prime}(\tau)$ field in Eq. 23. Next, we perform the local gauge transformation to the new bosonic variables

$$
\left[\begin{array}{c}
b(\tau) \\
\bar{b}_{i}(\tau)
\end{array}\right]=\left[\begin{array}{cc}
e^{i \phi_{i}(\tau)} & 0 \\
0 & e^{-i \phi_{i}(\tau)}
\end{array}\right]\left[\begin{array}{c}
a_{i}(\tau) \\
\bar{a}_{i}(\tau)
\end{array}\right]
$$

that removes the imaginary term $-i \int_{0}^{\beta} d \tau \dot{\phi}_{i}(\tau) n_{b i}(\tau)$ from all the Fourier modes except at zero frequency. From the above we deduce bosons have a composite nature made of bosonic part $a_{i}(\tau)$ and attached "flux" $\exp \left[i \phi_{i}(\tau)\right]$. Due to such U(1) gauge invariance, the fluctuations and the phase have the dynamics of $\mathrm{U}(1)$ gauge field.

\section{Gauge group U (1) governed phase only action}

By integrating out the auxiliary static field $V_{i 0}$ we calculate the partition function with an effective action expressed in the form of the propagator $\hat{G}$

$$
\mathcal{Z}=\int[\mathcal{D} \phi] e^{-\sum_{i} \int_{0}^{\beta} d \tau\left[\frac{1}{2 U_{\text {eff }}} \dot{\phi}_{i}^{2}(\tau)+\frac{1}{i} \frac{\bar{\mu}_{b}}{U_{\text {eff }}} \dot{\phi}_{i}(\tau)\right]+\operatorname{Tr} \ln \hat{G}^{-1}},
$$

where $\bar{\mu}_{b} / U_{b}=\mu_{b} / U_{b}+1 / 2$ is the shifted reduced bosonic chemical potential. In the above $\exp \left(-\operatorname{Tr} \ln \hat{G}^{-1}\right) \equiv$ $\operatorname{det} \hat{G}$ and the determinant takes the form

$$
\begin{aligned}
\operatorname{det} \hat{G} & =\int[\mathcal{D} \bar{a} \mathcal{D} a] \exp \left\{-\sum_{\langle i, j\rangle} \int_{0}^{\beta} d \tau\right. \\
& \times \bar{a}_{i}(\tau)\left[\left(\frac{\partial}{\partial \tau}+\bar{\mu}_{b}\right) \delta_{i j}\right. \\
& \left.\left.-e^{i \phi_{i}(\tau)} t_{b i j} e^{-i \phi_{j}(\tau)}\right] a_{i}(\tau)\right\} .
\end{aligned}
$$

We parametrize the boson fields $a_{i}(\tau)=a_{0}+a_{i}^{\prime}(\tau)$ and incorporate fully our calculations to the phase fluctuations governed by the gauge group U (1). Assuming nonfluctuating amplitude at low temperatures $a_{i}(\tau)=a_{0}$, we drop the corrections, which was proved to be justified in the large $U_{b} / t_{b}$ limit we are interested in [29, 32]. The amplitude fluctuations are massive one and do not play important role in the low energy scales. It is very convenient to define the order parameter

$$
\Psi_{\mathrm{B}} \equiv\left\langle b_{i}(\tau)\right\rangle=\left\langle a_{i}(\tau) \exp \left[i \phi_{i}(\tau)\right]\right\rangle=a_{0} \psi_{\mathrm{B}},
$$

which signals the emergence of the superfluid phase and vanishes in the Mott-insulator state. The SF state is characterized by spontaneously breaking of the $\mathrm{U}(1)$ symmetry of Bose-Fermi-Hubbard Hamiltonian. Note, that a nonzero value of the amplitude $a_{0}$ in Eq. 228 is not sufficient for superfluidity. To achieve this, also the phase variables $\phi$ in Eq. 28), must become stiff and coherent, which implies $\psi_{\mathrm{B}} \neq 0$. As we see in the next sections the presence of the fermions and density-density interactions $U_{b f}$ between species of different statistics can also change the amplitude of the order parameter. After mentioned assumption the inverse of the propagator becomes

$$
\hat{G}^{-1}=\hat{G}_{0}^{-1}-\hat{T}=\hat{G}_{0}^{-1}\left(1-\hat{T} \hat{G}_{0}\right) .
$$


The explicit value of the amplitude $a_{0}$ in Eq. 28 can be obtained from minimization of the Hamiltonian $\partial \mathcal{H}\left(a_{0}\right) / \partial a_{0}=0$. Therefore, we write

$$
\begin{aligned}
\hat{G}_{0} & =a_{0}^{2} \equiv \frac{\sum_{\langle i, j\rangle} t_{b i j}+\bar{\mu}_{b}}{U_{b}}-\frac{U_{b f}}{U_{b}} n_{\mathrm{F}} . \\
\hat{T} & =e^{i \phi_{i}(\tau)} t_{b i j} e^{-i \phi_{j}(\tau)} .
\end{aligned}
$$

Expanding the trace of the logarithm in Eq. (26) and making use the above we obtain up to the second order in the amplitude of the order parameter Eq. 28)

$$
\begin{aligned}
\operatorname{Tr} \ln \hat{G}^{-1} & =-\operatorname{Tr} \ln \hat{G}_{0}-\operatorname{Tr} \hat{T} \hat{G}_{0} \\
& -\frac{1}{2} \operatorname{Tr}\left(\hat{T} \hat{G}_{0}\right)^{2} .
\end{aligned}
$$

Trace over first term of the expansion, as previously, not containing any fluctuating field variables, gives an inessential constant contribution to the action. Let us consider the second order term in more detail

$$
\begin{aligned}
\operatorname{Tr}\left(\hat{T} \hat{G}_{0}\right) & =\sum_{\langle i, j\rangle} \tilde{t}_{b i j} \int_{0}^{\beta} d \tau d \tau^{\prime} \\
& \times e^{-i\left[\phi_{i}(\tau)-\phi_{j}\left(\tau^{\prime}\right)\right]} \delta\left(\tau-\tau^{\prime}\right),
\end{aligned}
$$

where

$$
\tilde{t}_{b i j}=a_{0}^{2} t_{b i j}=\left(\frac{\sum_{\langle i, j\rangle} t_{b i j}+\bar{\mu}_{b}}{U_{b}}-\frac{U_{b f}}{U_{b}} n_{f}\right) t_{b i j},
$$

the hopping matrix elements are re-normalized by the amplitude of the order parameter. We see that in comparison to pure bosonic case there is an additional shift $-U_{b f} n_{\mathrm{F}} / U_{b}$ that depends on the average of the fermion concentration and normalized inter-species interaction. The above was also obtained in the effective bosonic model and recognized as a mean-field contribution [9]. Finally, the partition function Eq. 26) becomes

$$
\mathcal{Z}=\int[\mathcal{D} \phi] e^{-\mathcal{S}_{\text {phase }}[\phi]}
$$

with an effective action expressed only in the phase fields variable

$$
\begin{aligned}
\mathcal{S}_{\text {phase }}[\phi] & =\int_{0}^{\beta} d \tau\left\{\sum_{i}\left[\frac{1}{2 U_{\text {eff }}} \dot{\phi}_{i}^{2}(\tau)+\frac{1}{i} \frac{\bar{\mu}_{b}}{U_{\text {eff }}} \dot{\phi}_{i}(\tau)\right]\right. \\
& \left.-\tilde{t}_{b} \sum_{i, j} e^{\phi_{i}(\tau)} I_{i j} e^{-\phi_{j}(\tau)}\right\}
\end{aligned}
$$

where $\tilde{t}_{b}=\left(\sum_{\langle i, j\rangle} t_{b i j}+\bar{\mu}_{b}-U_{b f} n_{\mathrm{F}}\right) t_{b} / U_{b}$. The total time derivative Berry phase imaginary term in Eq. (36) is nonzero due to topological phase field configurations with $\phi_{i}(\beta)-\phi_{i}(0)=2 \pi m_{i}\left(m_{i}=0, \pm 1, \pm 2 \ldots\right)$ that results in topological ingredients to the correlator we will see below. Therefore, we concentrate on closed paths in the "imaginary time" $(0, \beta)$ labeled by the integer winding numbers $m_{i}$. The path-integral

$$
\int[\mathcal{D} \phi] \ldots \equiv \sum_{\left[m_{i}\right]} \int_{0}^{2 \pi}[\mathcal{D} \phi(0)] \int_{\phi_{i}(0)}^{\phi_{i}(\tau)+2 \pi m_{i}}[\mathcal{D} \phi(\tau)] \ldots,
$$

includes a summation over $m_{i}$ and in each topological sector the integration goes over the gauge potentials. Therefore, we do not ignore the compactness of the gauge fields.

To proceed, we replace the phase degrees of freedom by the uni-modular scalar complex field $\psi$ which satisfies the quantum periodic boundary condition $\psi_{i}(\beta)=\psi_{i}(0)$. This can be conveniently done using the Fadeev-Popov method with Dirac delta functional resolution of unity, where we take $\psi$ as a continuous but constrained (on the average) variable to have the uni-modular value. We introduce

$$
\begin{aligned}
1 & =\int\left[\mathcal{D} \psi \mathcal{D} \psi^{*}\right] \delta\left(\sum_{i}|\psi(\tau)|^{2}-N\right) \\
& \times \delta\left(\psi_{i}-e^{i \phi_{i}(\tau)}\right) \delta\left(\psi_{i}^{*}-e^{-i \phi_{i}(\tau)}\right)
\end{aligned}
$$

and

$$
\begin{aligned}
\delta\left(\sum_{i}\left|\psi_{i}(\tau)\right|^{2}-N\right) & =\frac{1}{2 \pi i} \int_{-i \infty}^{+i \infty} d \lambda \\
& \times e^{\int_{0}^{\beta} d \tau \lambda\left(\sum_{i}\left|\psi_{i}(\tau)\right|^{2}-N\right)},
\end{aligned}
$$

where $N$ is the number of lattice sites. Introducing the Lagrange multiplier $\lambda$, which adds the quadratic terms (in the $\psi$ fields) to the action we can solve for the constraint. The partition function can be rewritten to the form

$$
\begin{aligned}
\mathcal{Z} & =\frac{1}{2 \pi i} \int_{-i \infty}^{+i \infty} e^{-\lambda N} d \lambda \int\left[\mathcal{D} \psi \mathcal{D} \psi^{*}\right] \\
& \times \exp \left\{-\sum_{i, j} \int_{0}^{\beta} d \tau d \tau^{\prime} \psi_{i}\left[\left(\tilde{t}_{b} I_{i j}+\lambda \delta_{i j}\right) \delta\left(\tau-\tau^{\prime}\right)\right.\right. \\
& \left.\left.+\gamma_{i j}\left(\tau, \tau^{\prime}\right)\right] \psi_{j}^{*}\right\}
\end{aligned}
$$

where

$$
\gamma_{i j}\left(\tau, \tau^{\prime}\right)=\left\langle\exp \left\{-i\left[\phi_{i}(\tau)-\phi_{j}\left(\tau^{\prime}\right)\right]\right\}\right\rangle
$$

is the two-point phase correlator associated with the order parameter field, where $\langle\cdots\rangle$ denotes averaging with respect to the action in Eq. (36). Because the values of the phases $\phi$ which differ by $2 \pi$ are equivalent we decompose phase field in terms of a periodic field and term linear in $\tau$ :

$$
\phi_{i}(\tau)=\varphi_{i}(\tau)+\frac{2 \pi}{\beta} m_{i} \tau
$$

with $\phi_{i}(\beta)=\phi_{i}(0)$. As a result the phase correlator factorizes as the product of a topological term depending on the integers $m_{i}$ and non-topological one:

$$
\gamma_{i j}\left(\tau, \tau^{\prime}\right)=\gamma_{i j}^{T}\left(\tau, \tau^{\prime}\right) \gamma_{i j}^{N}\left(\tau, \tau^{\prime}\right)
$$


Performing the Poisson re-summation formula in

$$
\gamma_{i j}^{T}\left(\tau, \tau^{\prime}\right)=\frac{\sum_{\left[m_{i}\right]} e^{-i \frac{2 \pi}{\beta}\left(\tau-\tau^{\prime}\right) m_{i}} e^{-\frac{2 \pi}{\beta} \sum_{i}\left[\frac{\pi}{U_{\text {eff }}} m_{i}^{2}+\frac{\beta}{i} \frac{\bar{\mu}_{b}}{U_{\text {eff }}} m_{i}\right]}}{\sum_{\left[m_{i}\right]} e^{-\frac{2 \pi}{\beta} \sum_{i}\left[\frac{\pi}{U_{\text {eff }}} m_{i}^{2}+\frac{\beta}{i} \frac{\bar{\mu}_{b}}{U_{\text {eff }}} m_{i}\right]}}
$$

and the functional integration over the phase variables

$$
\gamma_{i j}^{N}\left(\tau, \tau^{\prime}\right)=\frac{\int[\mathcal{D} \varphi] e^{-i\left[\varphi_{i}(\tau)-\varphi_{j}\left(\tau^{\prime}\right)\right]_{e}-\sum_{i} \frac{1}{2 U_{\mathrm{eff}}} \int_{0}^{\beta} d \tau \dot{\varphi}_{i}^{2}(\tau)}}{\int[\mathcal{D} \varphi] e^{-\sum_{i} \frac{1}{2 U_{\mathrm{eff}}} \int_{0}^{\beta} d \tau \dot{\varphi}_{i}^{2}(\tau)}}
$$

the final formula of the correlator takes the form

$$
\begin{aligned}
\gamma_{i j}\left(\tau, \tau^{\prime}\right) & =\frac{\vartheta\left(\pi \frac{\bar{\mu}_{b}}{U_{\text {eff }}}+\pi \frac{\tau-\tau^{\prime}}{\beta}, e^{-\frac{1}{U_{\text {eff }}} \frac{2 \pi^{2}}{\beta}}\right)}{\vartheta\left(\pi \frac{\bar{\mu}_{b}}{U_{\text {eff }}}, e^{-\frac{1}{U_{\text {eff }}} \frac{2 \pi^{2}}{\beta}}\right)} \\
& \times \exp \left(\frac{U_{\text {eff }}}{2}\left|\tau-\tau^{\prime}\right|-\frac{\left(\tau-\tau^{\prime}\right)^{2}}{\beta}\right),
\end{aligned}
$$

where $\vartheta(z, q)$ is the Jacobi theta function, which comes from the topological contribution - summation over integer winding numbers. The function $\vartheta(z, q)$ is defined by

$$
\vartheta(z, q)=1+2 \sum_{n=1}^{+\infty} \cos (2 n z) q^{n^{2}}
$$

and is $\beta$-periodic in the "imaginary time" as well in the variable $\bar{\mu}_{b} / U_{\text {eff }}$ with the period of unity which emphasizes the special role of its integer values. After Fourier transforming one obtains

$$
\gamma_{i j}\left(\omega_{\nu}\right)=\frac{1}{\mathcal{Z}_{0}} \frac{4}{U_{\text {eff }}} \sum_{\left[m_{i}\right]} \frac{e^{-\frac{U_{\text {eff }} \beta}{2} \sum_{i}\left(m_{i}+\frac{\bar{\mu}_{b}}{U_{\text {eff }}}\right)^{2}}}{1-4\left(\sum_{i} m_{i}+\frac{\bar{\mu}_{b}}{U_{\text {eff }}}-i \frac{\omega_{\ell}}{U_{\text {eff }}}\right)^{2}},
$$

where

$$
\mathcal{Z}_{0}=\sum_{\left[m_{i}\right]} e^{-\frac{U_{\text {eff }} \beta}{2} \sum_{i}\left(m_{i}+\frac{\bar{\mu}_{b}}{U_{\text {eff }}}\right)^{2}}
$$

is the partition function for the set of quantum rotors. The action Eq. (36), with the topological contribution Eq. 48, after Fourier transform, is written as

$$
\mathcal{S}_{\mathrm{eff}}[\psi, \bar{\psi}]=\frac{1}{N \beta} \sum_{\mathbf{k}, \ell} \bar{\psi}_{\mathbf{k}}\left(\omega_{\ell}\right) \Gamma_{\mathbf{k}}^{-1}\left(\omega_{\ell}\right) \psi_{\mathbf{k}}\left(\omega_{\ell}\right)
$$

where $\Gamma_{\mathbf{k}}^{-1}\left(\omega_{\ell}\right)=\lambda-t_{b \mathbf{k}}+\gamma^{-1}\left(\omega_{\ell}\right)$ is the inverse of the propagator and $t_{b \boldsymbol{k}}$ is the Fourier transform of the bosonic hopping matrix elements for two- $t_{b \boldsymbol{k}}^{2 D}=2 t_{b}\left(\cos k_{x}+\cos k_{y}\right)$ and three-dimensional $t_{b \boldsymbol{k}}^{3 D}=$ $2 t_{b}\left(\cos k_{x}+\cos k_{y}+\cos k_{z}\right)$ lattice.

\section{CRITICAL LINE}

Within the phase coherent state the order parameter $\psi_{B}$ is evaluated in the thermodynamic limit $N \rightarrow \infty$ by the saddle point method $\delta \mathcal{F} / \delta \lambda=0$ and the uni-modular condition of the $\mathrm{U}(1)$ phase variables translates into the equation

$$
1-\psi_{\mathrm{B}}^{2}=\lim _{N \rightarrow \infty} \frac{1}{N \beta} \sum_{\mathbf{k}, \ell} \Gamma_{\boldsymbol{k}}\left(\omega_{\ell}\right)
$$

with

$$
\Gamma_{\boldsymbol{k}}^{-1}\left(\omega_{\ell}\right)=\tilde{t}_{b \mathbf{k}=0}-\tilde{t}_{b \mathbf{k}}+\frac{1}{U_{\mathrm{eff}}} \bar{\mu}_{b}^{2}-\frac{1}{U_{\mathrm{eff}}}\left(\bar{\mu}_{b}-i \omega_{\ell}\right)^{2} .
$$

The phase boundary is determined by the divergence of the order parameter susceptibility $\Gamma_{\mathbf{k}=0}\left(\omega_{\ell=0}\right)=0$

$$
\lambda_{0}-t_{b \boldsymbol{k}=0}^{\max }+\gamma^{-1}\left(\omega_{\ell=0}\right)=0
$$

which determines the critical value of the Lagrange parameter $\lambda=\lambda_{0}$ and stays constant in the whole global coherent phase. To proceed, it is desirable to introduce the density of states

$$
\rho(\xi)=\frac{1}{N} \sum_{\boldsymbol{k}} \delta\left(\xi-\frac{t_{b \boldsymbol{k}}}{t_{b}}\right)
$$

because the analytical expressions we use can be advantageous in evaluating sums over momenta. The corresponding formulas for square lattice can be written as

$$
\rho^{2 D}(\xi)=\frac{1}{2 \pi^{2} t_{b}} \mathbf{K}\left(\sqrt{1-\left(\frac{\xi}{4 t_{b}}\right)^{2}}\right) \Theta\left(1-\left|\frac{\xi}{4 t_{b}}\right|\right),
$$

and for simple cubic geometry takes form

$$
\begin{aligned}
\rho^{3 D}(\xi) & =\frac{1}{2 \pi^{3} t_{b}} \int_{a_{1}}^{a_{2}} \frac{d \epsilon}{\sqrt{1-\epsilon^{2}}} \Theta\left(1-\frac{|\xi|}{6 t_{b}}\right) \\
& \times \mathbf{K}\left(\sqrt{1-\left(\frac{\xi}{4 t_{b}}+\epsilon\right)^{2}}\right)
\end{aligned}
$$

with $a_{1}=\min \left(-1,-2-\xi / 2 t_{b}\right)$ and $a_{2}=$ $\max \left(1,2-\xi / 2 t_{b}\right) ; \mathbf{K}(x)$ is the elliptic function of the first kind. 33. After summation over Bose-Matsubara frequency and for zero temperature limit $\beta \rightarrow \infty$ we can rewrite the critical line equation to the form that represents solution of the BF Hubbard model in terms of re-normalized pure Bose-Hubbard Hamiltonian in the quantum rotor approach: 


$$
1-\psi_{\mathrm{B}}^{2}=\frac{1}{2} \int_{-\infty}^{+\infty} \frac{\rho(\xi) d \xi}{\sqrt{2\left(\xi_{\max }-\xi\right)\left(2 z \frac{t_{b}}{U_{b}}+\frac{\mu_{b}}{U_{b}}-\eta+\frac{1}{2}\right) \frac{1}{\alpha} \frac{t_{b}}{U_{b}}+v^{2}\left(\frac{1}{\alpha} \frac{\mu_{b}}{U_{b}}\right)}}
$$

In the above $v\left(\mu_{b} / \alpha U_{b}\right)=\operatorname{frac}\left(\mu_{b} / \alpha U_{b}\right)-1 / 2$, where frac $(x)=x-[x]$ is the fractional part of the number and $[x]$ is the floor function which gives the greatest integer less than or equal to $x$; $\xi_{\max }$ stands for the maximum value of the bosonic dispersion spectrum $t_{b k}$ and $z$ is the lattice coordination number. The renormalization parameters are defined as:

$$
\begin{aligned}
\alpha & =1+\frac{U_{b f}^{2}}{U_{b}} \chi^{\prime}\left(\omega, \mu_{f}\right) \\
\eta & =\frac{U_{b f}}{U_{b}} n_{\mathrm{F}}
\end{aligned}
$$

and allow us to see how adding free fermions to strongly interacting bosons confined in optical lattice influences the phase boundary.

\section{PHASE DIAGRAMS - BH MODEL}

The zero-temperature phase diagram of the BoseFermi-Hubbard model Eq. (1) can be calculated from Eq. (57) and usually is plotted as a function of $t_{b} / U_{b}$, with the density of the bosons controlled by a chemical potential $\mu_{b} / U_{b}$. The presence of the fermions implicates two additional different parameters that can by varied namely $\alpha$ and $\eta$ in Eq. (58) and Eq. (59). The strength of the inter-species interaction influences both of them, however the sign of $U_{b f}$ and the average density of fermions $n_{\mathrm{F}}$ added to the system affects only $\eta$. Besides, in the local dynamic approach the sign of the density-density interaction depends also on the normalized frequency $\omega / t_{f}$. For a general choice of parameters, Eq. (57) is easy to solve, however considerations of special cases can provide more insights into the solution of the problem. In discussion we will follow the scheme

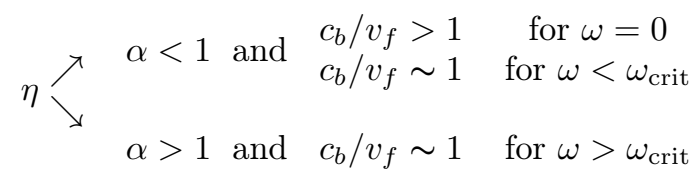

firstly choosing the sign of the $\eta$ and later $\alpha$ in the static $(\omega=0)$ or dynamic $(\omega \neq 0)$ limit (see Fig. 1).

Before we proceed with analysis let us introduce the notation for the maximum of the critical value for parameter $t_{b} / U_{b}$ (as a function of the normalized chemical potential $\left.\mu_{b} / U_{b}\right)$ at the tip of the $n$th $\left(n_{\mathrm{B}}=n\right)$ MI lobe for different lattice geometries and model parameters $\alpha$
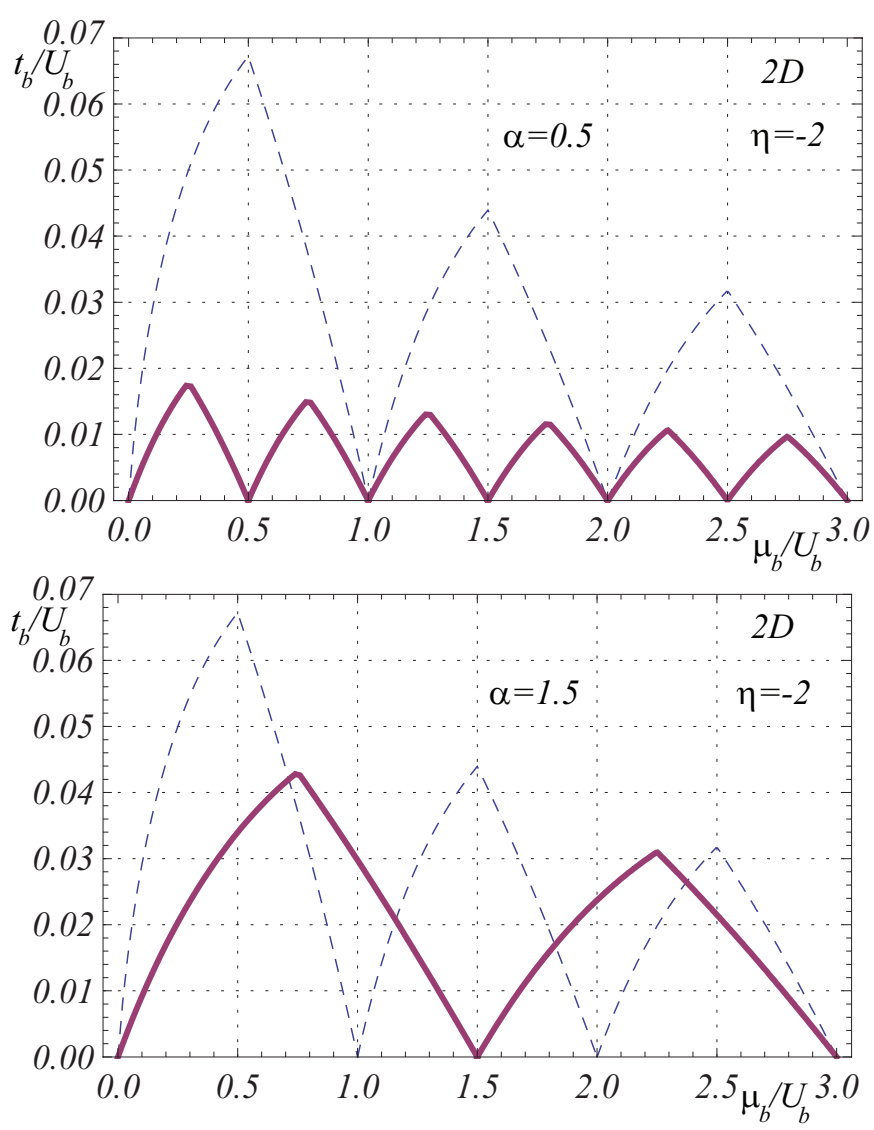

Figure 4: (Color online) Phase diagrams $\left(t_{b} / U_{b}-\mu_{b} / U_{b}\right)$ for the square $(2 D)$ lattice for different $\alpha=0.5$ (higher panel, $\omega<$ $\left.\omega_{\text {crit }}\right), \alpha=1.5\left(\omega>\omega_{\text {crit }}\right)$ and $\eta=-2$ (negative scattering length $\left.a_{b f}<0\right)$. Dashed line stands for the phase boundary of one-component Bose-Hubbard model. Within the lobes the Mott insulator phase takes place with $\Psi_{\mathrm{B}}=0$.

and $\eta$ as follows

$$
x_{n}(\alpha, \eta) \equiv \max \left\{\left(\frac{t_{b}}{U_{b}}\right)_{\text {crit }}\right\}_{\alpha, \eta}^{2 D, 3 D} .
$$

The above determines when the transition from MI to SF occurs. Values $\alpha=1$ and $\eta=0$ stand for the onecomponent bosonic case. In Table I we show comparison of $x_{n}(1,0)$ for higher densities of the particles calculated in the quantum rotor approach (QRA) to very accurate, recently developed, diagrammatic perturbation approach 34] to Bose-Hubbard Hamiltonian. The results for $3 D$ BH model obtained in both theories are very close and also comparison to quantum Monte-Carlo 

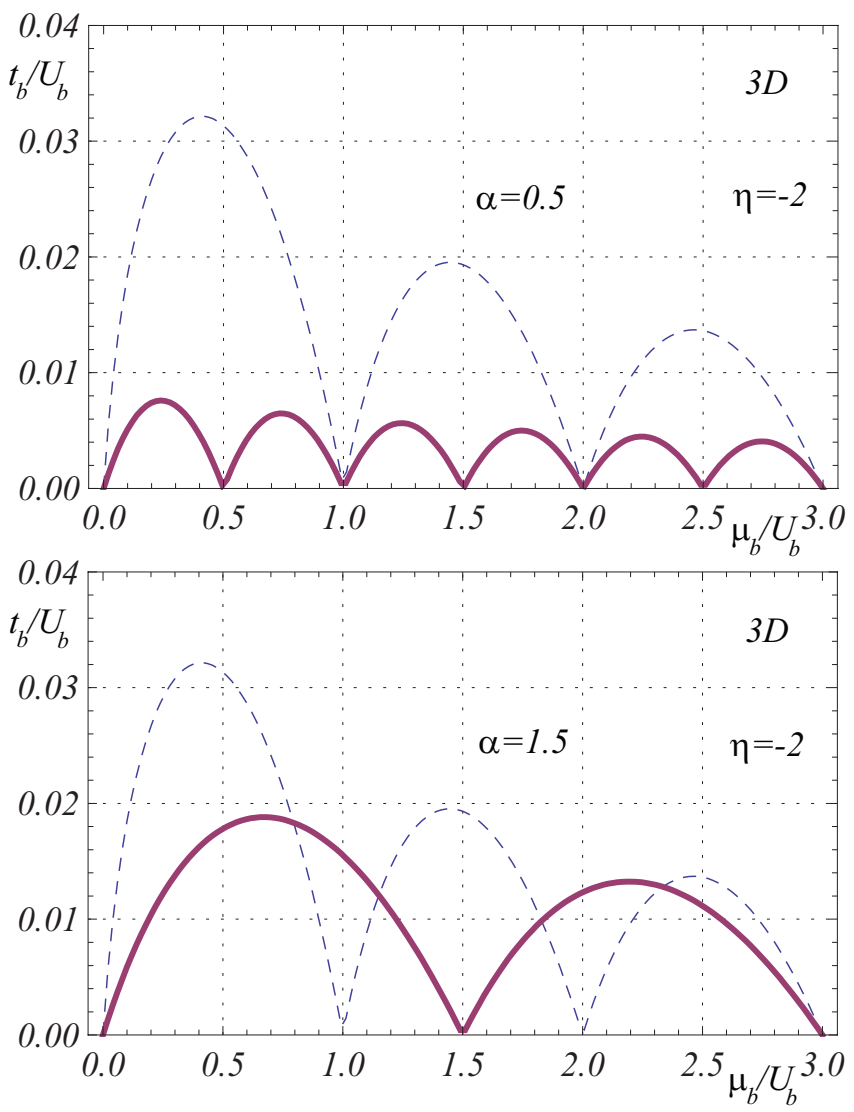

Figure 5: (Color online) Phase diagrams $\left(t_{b} / U_{b}-\mu_{b} / U_{b}\right)$ for the cubic $(3 D)$ lattice for different $\alpha=0.5$ (higher panel, $\omega<$ $\left.\omega_{\text {crit }}\right), \alpha=1.5\left(\omega>\omega_{\text {crit }}\right)$ and $\eta=-2$ (negative scattering length $\left.a_{b f}<0\right)$. Dashed line stands for the phase boundary of one-component Bose-Hubbard model. Within the lobes the Mott insulator phase takes place with $\Psi_{\mathrm{B}}=0$.

(QMC) numerical calculations 35 indicates that methods we use are able to properly catch the interesting physics of strongly interacting systems. However, we want to analyze the phase boundary for number of particles per lattice sites higher than one $n_{\mathrm{B}}>1$ that adds another dimension to the analysis and is difficult for the QMC to catch. The phase boundary for square lattice shows that QRA works well also in low-dimensional geometries, especially for higher densities. Nevertheless, the structure of Eq. (57) can cause some problems when both $\alpha$ and $\eta$ are nonzero and the number of bosons is equal one per lattice sites. We expect that for $\eta>1$ some artificial effects may arise for values of the normalized chemical potential $\mu_{b} / U_{b} \approx 0$ close to zero.

\section{BOSE-FERMI-HUBBARD PHASE DIAGRAM}

In the experiments 4 for a degenerate mixtures of $4 \times 10^{5}{ }^{87} \mathrm{Rb}$ bosons and $3 \times 10^{5}{ }^{40} \mathrm{~K}$ fermions the scattering length $a_{b f}$ (and in consequence interaction

\begin{tabular}{c|c|c|c|c|c}
$2 D$ & $n_{\mathrm{B}}=1$ & 2 & 3 & 4 & 10 \\
\hline DPT & 0.0590934 & 0.0348009 & 0.0247350 & 0.0191986 & 0.0082079 \\
\hline QRA & 0.0671998 & 0.0439387 & 0.0317523 & 0.0246185 & 0.0093296 \\
\hline $3 D$ & $n_{\mathrm{B}}=1$ & 2 & 3 & 4 & 10 \\
\hline DPT & 0.0340685 & 0.0200755 & 0.0142709 & 0.0110779 & 0.0047362 \\
\hline QRA & 0.0321429 & 0.0194846 & 0.0136102 & 0.0103755 & 0.0042086
\end{tabular}

Table I: Comparison of the maximum of the critical value for $\left(t_{b} / U_{b}\right)_{\text {crit }}$ (as a function of the normalized bosonic chemical potential $\left.\mu_{b} / U_{b}\right)$ at the tip of the $n$th $\left(n_{\mathrm{B}}=1 \div 4\right.$ and 10) Mott insulator lobe for the square $(2 D)$ and cubic $(3 D)$ lattice in the one-component Bose-Hubbard model: DPT - diagrammatic perturbation theory [34]), QRA - our calculations using quantum rotor approach).
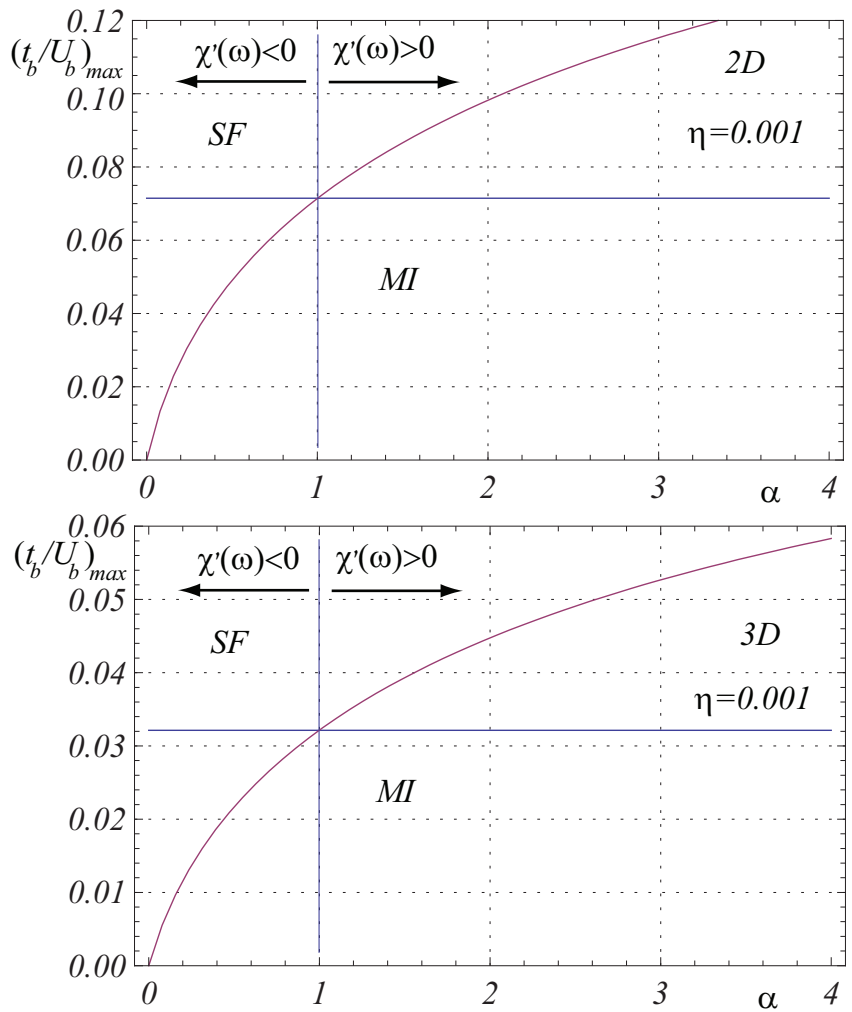

Figure 6: (Color online) Maximum value of the $\left(t_{b} / U_{b}\right)_{\max }$ for different $\alpha$ and $\eta=0.001$ (very small amount of fermions) for square $(2 D)$ and cubic $(3 D)$ lattice with $n_{\mathrm{B}}=1$. Vertical solid line stands for the $\left(t_{b} / U_{b}\right)_{\max }$ obtained in one-component Bose-Hubbard model with one particle per lattice site. Above the curves the superfluid phase takes place with $\Psi_{\mathrm{B}} \neq 0$.

$U_{b f}$, see Eq. (4) can be continuously tune between $-170 a_{0} \div+800 a_{0}$ below and between $-800 a_{0} \div-200 a_{0}$ above Feshbach resonance, where $a_{0}$ is the Bohr radius. The form of the parameters we choose Eq. (58) and Eq. (59) allows for its interpretation. The periodicity of the phase diagram can be easily deduced from the periodic properties of the propagator Eq. (48) and strongly depends on $\alpha$ however, the interaction between species does not generate additional Mott lobes in the phase diagram. 
The above is in contrast to the strong coupling expansion and exact diagonalization method applied to the system of two alkali-metal atoms with different masses where (for very small lattice sizes and quenched disorder) the MI phases with integer filing factors disappear for boson-impurity interaction energy larger than on-site atom-atom interaction energy itself and also the MI phase exists for incommensurate bosonic filling [25]. If we fix the number of fermions $n_{\mathrm{F}}$ and inter-species interaction $U_{b f}$ in Eq. (59) still there is a dynamic part of the local Lindhard function $\chi^{\prime}\left(\omega, \mu_{f}\right)$ we have to take into account. In the static limit $\omega / t_{f} \rightarrow 0$ (where the Lindhard response function is purely real) there is nothing unexpected in the behavior of the critical line (see discussion below). However, we must stress that even we left the frequency dependence apart, there is still very interesting part of physics remained, because the Lindhard response function for the system with regular density of states shows logarithmic divergence as temperature goes to zero. These singularities give rise to instabilities in the system towards two new ground states a phase separated state or a supersolid phase [11, 36. On the other hand the oscillation of the induced effective interaction between bosons is the origin of the formation of charge density waves [9].

Taking $\omega<\omega_{\text {crit }}$ we recover the previous theoretical results where, after adding fermions to the system, the effective interaction $U_{\text {eff }}$ becomes smaller than repulsive energy $U_{b}$ for bosons only (see Fig. 4 and Fig. 5 ) and superfluid phase increases. The above is best shown on Fig. 6] where for very small amount of fermions $n_{\mathrm{F}}$ the parameter $\alpha<1$ causes decreasing the Mott insulator region of the phase diagram in comparison to the pure bosonic case. However, in the local dynamic limit, when $\omega>\omega_{\text {crit }}$ the Mott insulator phase is becoming stronger and bosons tend to localize on the lattice sites in both $2 D$ and $3 D$ cases (see Fig. 6, Fig. 7 and Fig. 8).

One may argue that the Lindhard response function depends not only on the frequency $\omega / t_{f}$ but also on the chemical potential $\mu_{f} / t_{f}$ for fermions and so far we did not restrict ourselves to any particular value of it. In many approaches it is a little tricky to handle because despite of the absence of any direct interaction between fermions $U_{f f}=0$ the density-density fluctuations can indeed induce some effective interaction between fermionic species [37]. Therefore problem becomes complex and many theories just take half-filled band with $n_{\mathrm{F}}=1$ so that $\mu_{f} / t_{f}=0$. Alternative approach comes from partial particle-hole symmetry Hamiltonian Eq. (1) possesses. To make our approach self-consistent we can calculate how does a particular value of the fermionic chemical potential change in the effectively interacting system. We remind that the amplitude of the order parameter was obtained from minimization condition, assuming nonfluctuating bosonic amplitude at low temperatures. By operating a similar procedure we get a shift of the chemical
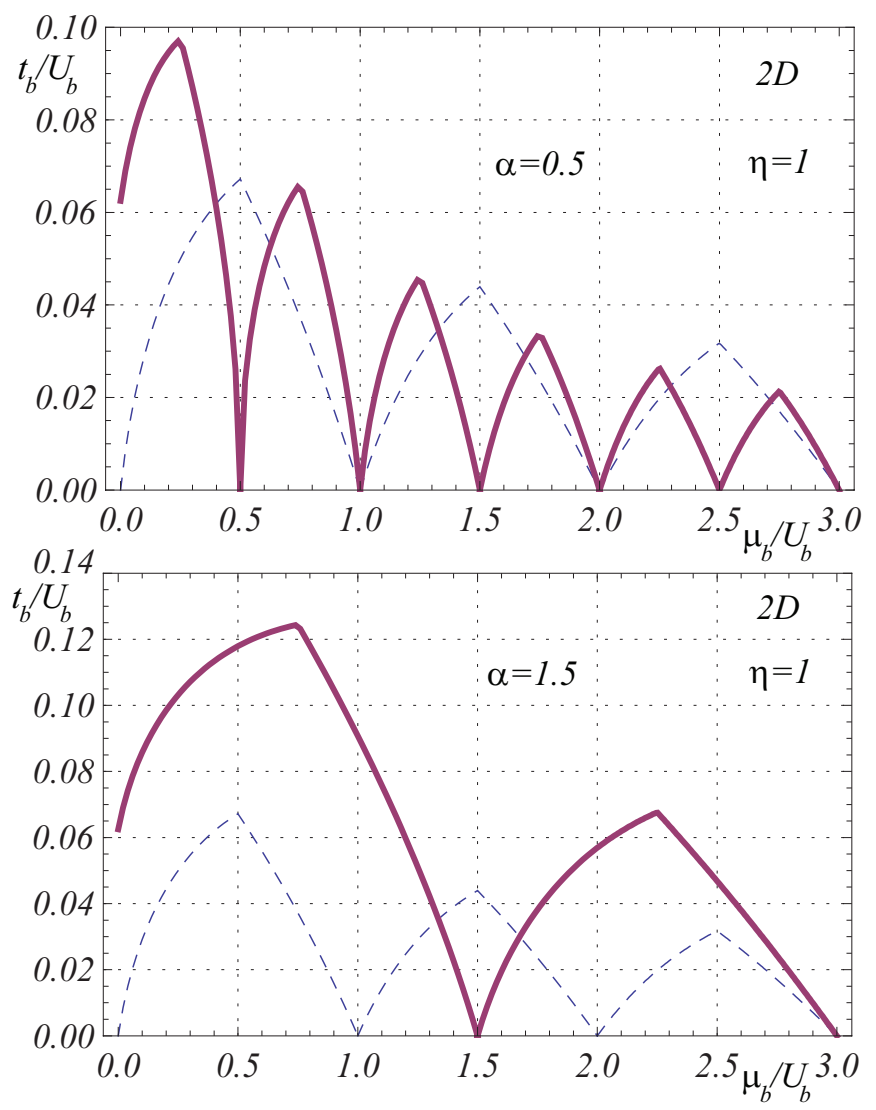

Figure 7: (Color online) Phase diagrams $\left(t_{b} / U_{b}-\mu_{b} / U_{b}\right)$ for the square $(2 D)$ lattice for different $\alpha=0.5$ (higher panel, $\left.\omega<\omega_{\text {crit }}\right), \alpha=1.5\left(\omega>\omega_{\text {crit }}\right)$ and $\eta=1$ (positive scattering length $\left.a_{b f}>0\right)$. Dashed line stands for the phase boundary of one-component Bose-Hubbard model. Within the lobes the Mott insulator phase takes place with $\Psi_{\mathrm{B}}=0$.

potential for fermions

$$
\mu_{f} \rightarrow \mu_{f}-U_{b f}\left(2 z \frac{t_{b}}{U_{b}}+\frac{\mu_{b}}{U_{b}}-\eta+\frac{1}{2}\right),
$$

that in the non-interacting case $U_{b f}=0$ reduces to that of free particles obeying fermionic commutation relations (see also Appendix).

There is the limit where the system containing gaseous mixtures has the same value of $x(\alpha, \eta)$ as in the case of only bosons confined in optical lattice (see Fig. 9p. Again, we take advantage of the choice of the parameters, that suits well our goal, and make notation of the condition very simple. If $\eta=1-\alpha$ we have

$$
x_{n}(1,0)=x_{n}(\alpha, 1-\alpha) .
$$

The above, in terms of the original variables, leads to $U_{b f} n_{\mathrm{F}} / U_{b}=U_{b f}^{2} \chi^{\prime}\left(\omega, \mu_{f}\right) / U_{b}$. For cubic lattice the formula Eq. (63) seems to not hold (Fig. 9) however is accurate with a numerical error less than 0.17 percent. Therefore, if the number of fermions added to the system is equal to the inter-species interaction then bosons behaves as if were unaffected by the presence of fermions. 

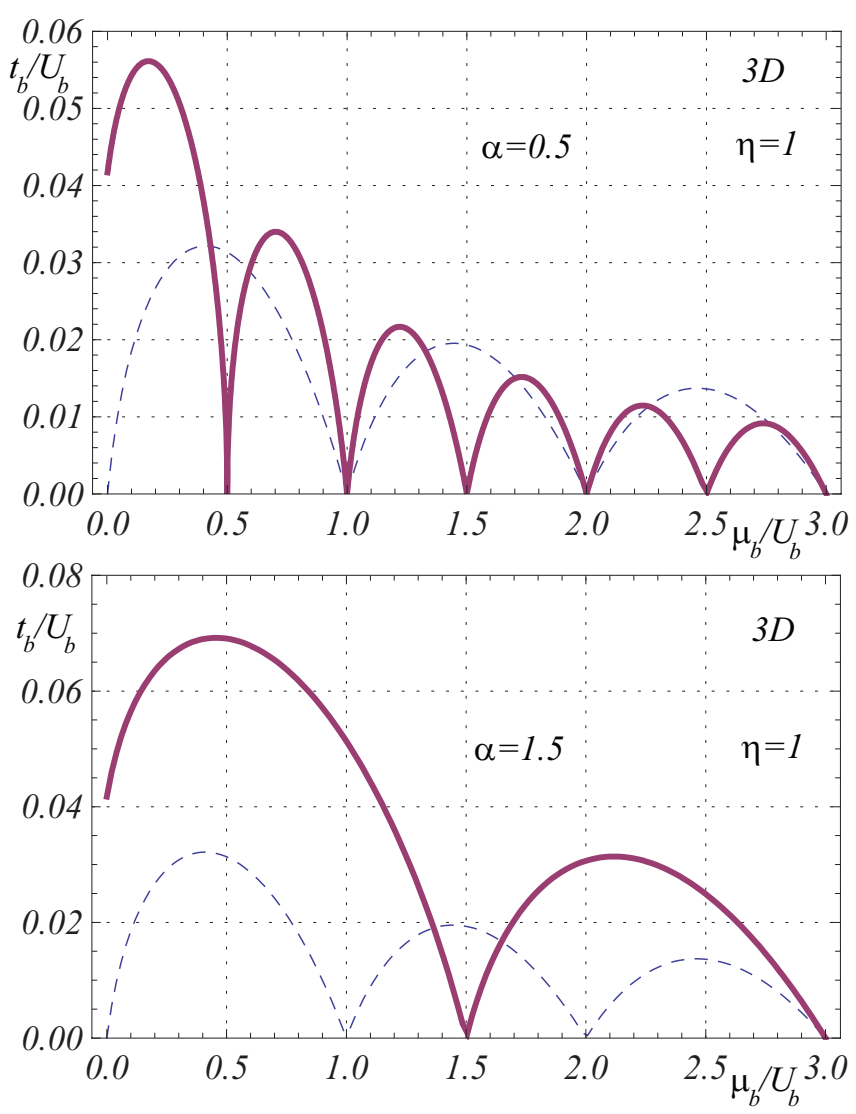

Figure 8: (Color online) Phase diagrams $\left(t_{b} / U_{b}-\mu_{b} / U_{b}\right)$ for the cubic $(3 D)$ lattice for different $\alpha=0.5$ (higher panel, $\left.\omega<\omega_{\text {crit }}\right), \alpha=1.5\left(\omega>\omega_{\text {crit }}\right)$ and $\eta=1$ (positive scattering length $\left.a_{b f}>0\right)$. Dashed line stands for the phase boundary of one-component Bose-Hubbard model. Within the lobes the Mott insulator phase takes place with $\Psi_{\mathrm{B}}=0$.

As a matter of fact we have to remember about sign of the scattering length $a_{b f}$ and normalized frequency $\omega / t_{f}$ that also modifies the introduced condition. The recent experiment 4] shows that there is an asymmetry in profiles of visibility of the interference pattern (recorded by absorption imaging) versus the inter-species scattering length that increases with lattice depth. Presented data indicate that visibility shows a maximum at the position consistent with $a_{b f}=0$. Besides, there is an asymmetry in a shift of the MI to SF transition boundary. Our calculations can reproduce latter however if the sign of the inter-species interaction is negative the MI phase diminishes and quite oppositely for positive scattering length and, as we expected, some anomaly appears at the point with $\mu_{b} / U_{b}=0$ (see Fig. 7 and Fig. 8). There is no physical reason for the phase boundary to change a position where the chemical potential for bosons is zero and the repulsive interactions are very strong $U_{b} \rightarrow \infty$. Moreover, in that case the value obtained from Eq. (57) at mentioned point $\left(\mu_{b} / U_{b}=0\right.$ and fixed $\left.\eta>0\right)$ is constant in whole nonzero range of the parameter $\alpha$ and depends only on the considered topology of the system. The
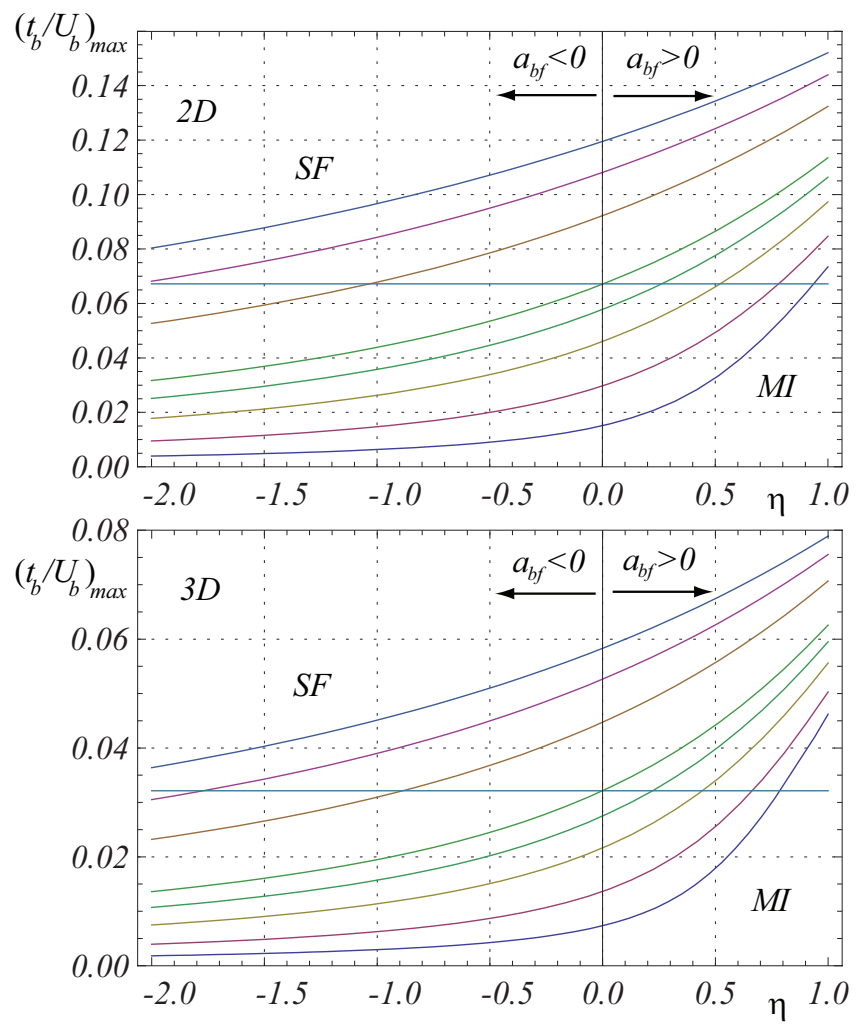

Figure 9: (Color online) The maximum of the critical value for the parameter $\left(t_{b} / U_{b}\right)_{\max }$ with $n_{\mathrm{B}}=1$ for different $\alpha=4$, $3,2,1,0.75,0.5,0.25,0.1$ for square $(2 D)$ and cubic $(3 D)$ lattice. Vertical solid line stands for the $\left(t_{b} / U_{b}\right)_{\max }$ obtained in the one-component Bose-Hubbard model with one particle per lattice site. Above the curves the superfluid phase takes place with $\Psi_{\mathrm{B}} \neq 0$.

similar to fermion-boson loss of coherence for the bosonboson species was found using the Gutzwiller mean-field approach [27]. The main effect of the addition different species of the same statistics is that the new structure of wedding cake appear but the oscillatory behavior of the relevant condensate fraction does not necessarily result in increase of the overall coherence of other species. The later is limited exclusively to the shallow lattice depth and was never observed in the experiments.

We want to stress that one have to be careful with the analysis of the phase diagrams. The summary of our results for square lattice (we omit qualitatively similar results for cubic geometry) is presented on Fig (10) and Fig (11). For the static and dynamic limit, but below the critical frequency $\omega<\omega_{\text {crit }}$ the Mott insulator region on the phase diagram broadens only when the scattering length is positive (the part of the surface above the plane of the critical value of $x_{1}(1,0)$ for the one-component $\mathrm{BH}$ model). When we must take into account the difference in the inter-species masses $m_{b} / m_{f} \neq 1$ the sign of the real part of the local Lindhard response function may become positive and, in consequence, $\alpha$ parameter takes values above one Fig. (11) leading to higher repulsive 


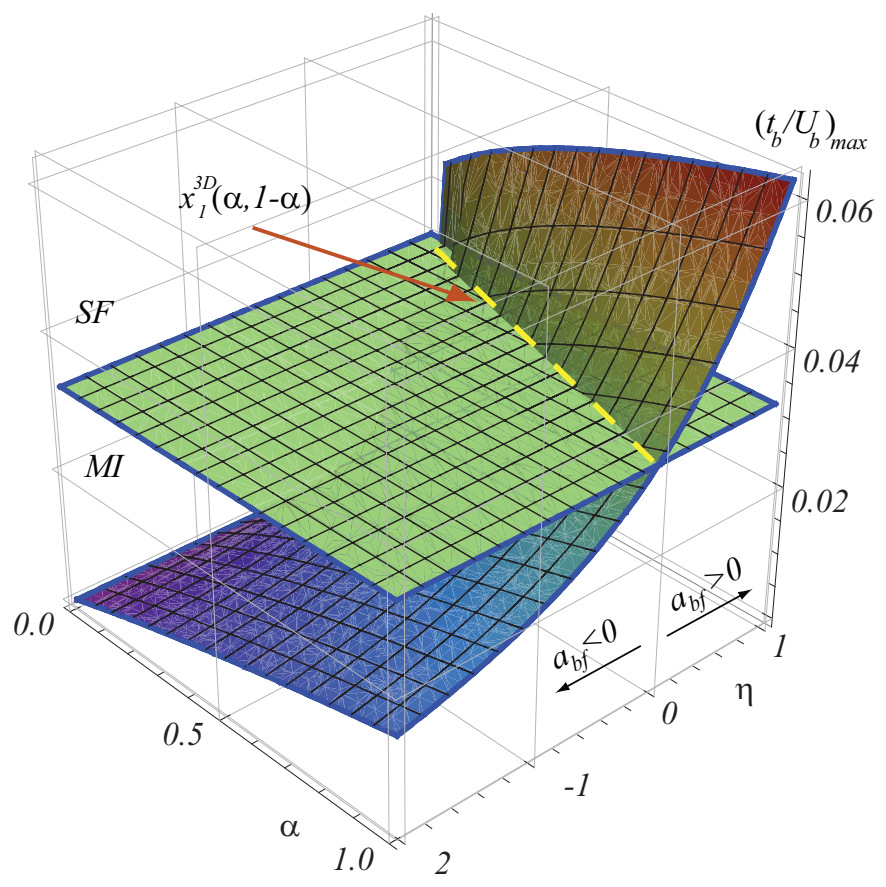

Figure 10: (Color online) The maximum of the critical value for the parameter $\left(t_{b} / U_{b}\right)_{\max }$ with the negative real part of the local dynamic Lindhard function $\chi^{\prime}(\omega)<0\left(\omega<\omega_{\text {crit }}\right)$, for cubic $(3 D)$ lattice in the space of the dimensionless parameters $\alpha-\eta$ with $n_{\mathrm{B}}=1$. The flat surface stands for $x_{1}^{3 D}(1,0)$ (see also Eq. 63) in case of the one-component BoseHubbard model with one particle per lattice site. The dashed line stands for the condition where the system of gaseous mixtures has the same value $\left(t_{b} / U_{b}\right)_{\max }$ as only bosons confined in optical lattice. Above the surfaces the superfluid phase takes place with $\Psi_{\mathrm{B}} \neq 0$.

energy between bosons even if measured scattering length is negative. In that case for $\alpha>1-\eta$ there is always a shift for higher values for the parameter $x_{n}(\alpha, \eta>1-\alpha)$ results in stronger localization of the bosons after adding fermions to the system.

\section{CONCLUSIONS}

It is well known that the ground state of a system of repulsively interacting bosons confined in a periodic potential can be either in a superfluid or in a Mott-insulting state, characterized by integer boson densities. Because the phase of the order parameter and the particle number, as conjugate variables, are subject to the uncertainty principle $\Delta \phi \Delta n \sim \hbar$, so the bosons can either be in the eigenstate of particle number or phase. The eigenstate of phase is a superfluid and that of particle number is a localized Mott insulator. Therefore, the quantum MISF phase transition takes place as the particle density is shifted thus facilitating emergence of the superfluid from the Mott insulating state. Adding to bosons particles of different statistics and allowing for the mutual repulsion

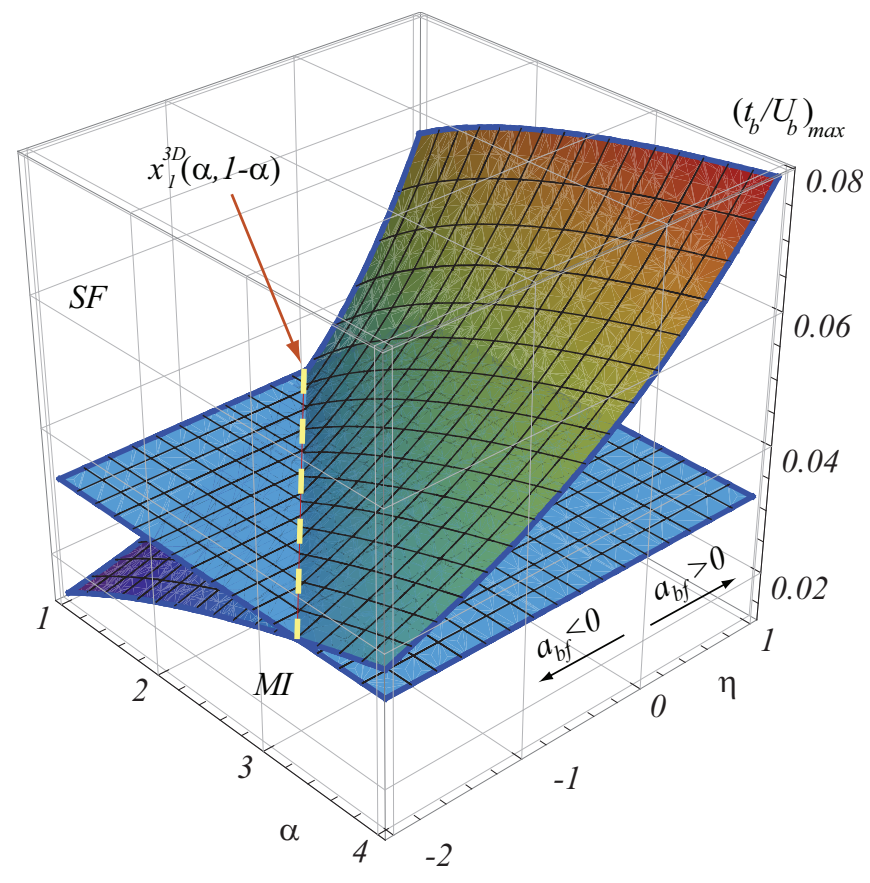

Figure 11: (Color online) The maximum of the critical value for the parameter $\left(t_{b} / U_{b}\right)_{\max }$ with the positive value of the real part of the local dynamic Lindhard function $\chi^{\prime}(\omega)>0$ $\left(\omega>\omega_{\text {crit }}\right)$, for cubic $(3 D)$ lattice in the space of the dimensionless parameters $\alpha-\eta$ with $n_{\mathrm{B}}=1$. The flat surface stands for $x_{1}^{3 D}(1,0)$ (see also Eq. 63) in case of the onecomponent Bose-Hubbard model with one particle per lattice site. The dashed line stands for the condition where the system of gaseous mixtures has the same value $\left(t_{b} / U_{b}\right)_{\max }$ as only bosons confined in optical lattice. Above the surfaces the superfluid phase takes place with $\Psi_{\mathrm{B}} \neq 0$.

or attraction between species strongly affects the equilibrium properties. We presented a field-theoretic study of the ground-phase diagram in quantum two- and threedimensional gaseous Bose-Fermi condensates where mentioned emulation takes place. We calculated the phase diagram using the quantum rotor approach that can reproduce the asymmetry in a shift of the MI to SF transition boundary for positive and negative inter-species scattering length. Analysis of the local dynamic Lindhard function revealed the critical value of the frequency for the collective excitations, where the real part of the response function (and in consequence the interaction between bosons and fermions) alters sign. The choice of the parameters of the model led to simple condition for the experimentally accessible parameters within the phase diagram for Bose-Fermi mixtures is qualitatively the same as for one-component repulsively interacting Bose system. We also compared the maximum of the critical value for $t_{b} / U_{b}$ parameter (as a function of the normalized chemical potential $\mu_{b} / U_{b}$ ) at the tip of the $n$th MI lobe for square and cubic lattice with numerical diagrammatic method and found them in a good agreement especially for higher, experimentally realizable, fill- 
ing factors. The nice feature of presented approach, described in details above, is that all the expressions and handling are analytic. It is also worth to notice that provided local approximation can be very useful in various situations whenever the retardation effects has to be taken into account and we are not interested in effects caused by non-locality.

\section{Appendix A: Local dynamical approach}

The third term of the trace (Eq. 14) can be written after Fourier transform in form

$$
\begin{aligned}
\operatorname{Tr} \ln \hat{G}_{f+\mathrm{int}}^{-1} & =\sum_{\boldsymbol{k} \boldsymbol{k}^{\prime}, \ell, \ell^{\prime}} \frac{\bar{b}_{\boldsymbol{k}-\boldsymbol{k}^{\prime}}\left(\omega_{\ell}-\omega_{\ell^{\prime}}\right) b_{\boldsymbol{k}-\boldsymbol{k}^{\prime}}\left(\omega_{\ell}-\omega_{\ell^{\prime}}\right)}{t_{f \boldsymbol{k}}+i \nu_{\ell}} \\
& \times \frac{\bar{b}_{\boldsymbol{k}-\boldsymbol{k}^{\prime}}\left(\omega_{\ell^{\prime}}-\omega_{\ell}\right) b_{\boldsymbol{k}-\boldsymbol{k}^{\prime}}\left(\omega_{\ell^{\prime}}-\omega_{\ell}\right)}{t_{f \boldsymbol{k}^{\prime}}+i \nu_{\ell^{\prime}}} \\
& =\sum_{\boldsymbol{q}} \chi\left(\boldsymbol{q}, i \tilde{\nu}_{\ell}, \mu_{f}, \beta\right) \\
& \times \sum_{\ell} \Lambda_{\boldsymbol{q}}\left(\omega_{\ell}\right) \Lambda_{-\boldsymbol{q}}\left(-\omega_{\ell}\right) .
\end{aligned}
$$

In the above we picked up some special value of the frequency $\tilde{\nu}_{\ell}$. Now, doing the inverse Fourier transform and using gradient expansion

$$
b_{j}\left(\tau^{\prime}\right)=b_{i}(\tau)+\left(\tau-\tau^{\prime}\right) \partial_{\tau} b_{j}(\tau)+\mathcal{O}\left[\left(\tau-\tau^{\prime}\right)^{2}\right]
$$

we obtain local, in the Matsubara-imaginary time, quadratic form of the trace in the bosonic variables

$$
\operatorname{Tr} \ln \hat{G}_{f+\mathrm{int}}^{-1} \rightarrow \chi^{\prime}\left(i \tilde{\nu}_{\ell}, \mu_{f}, \beta\right) \sum_{i} \int_{0}^{\beta} d \tau\left[\bar{b}_{i}(\tau) b_{i}(\tau)\right]^{2}
$$

We performed an expansion were not the $b_{j}\left(\tau^{\prime}\right)$ degree of freedom itself but rather its gradients of $\partial_{\tau} b_{j}(\tau)$ are assumed to be small. The explicit formula of the imaginary part for the dynamical Lindhard function is calculated in the next section.

\section{Appendix B: Local (momentum integrated) Lindhard function}

To stay in the local regime and using an analytic continuation $i \nu_{\ell} \rightarrow \omega+i \epsilon$, where $i \epsilon$ comes from the causality relation of the response function

$$
\lim _{\epsilon \rightarrow 0^{+}} \frac{1}{\omega \pm i \epsilon}=\mathcal{P}\left(\frac{1}{\omega}\right) \pm i \pi \delta(\omega)
$$

where symbol $\mathcal{P}$ denotes the Cauchy principal value which prevents divergence when $\omega=\omega^{\prime}$, we calculate the explicit value of the imaginary part that is somewhat easier to obtain than the real part

$$
\begin{aligned}
\chi^{\prime \prime}\left(i \nu_{\ell} \rightarrow \omega+i \epsilon, \mu_{f}, \beta\right) & =\sum_{\mathbf{k}, \boldsymbol{q}} \frac{f\left[t_{f \boldsymbol{k}}, \mu_{f}, \beta\right]-f\left[t_{f \boldsymbol{k}+\boldsymbol{q}}, \mu_{f}, \beta\right]}{t_{f \boldsymbol{k}}-t_{f \boldsymbol{k}+\boldsymbol{q}}-\omega+i \epsilon} \\
& =\lim _{\epsilon \rightarrow 0^{+}} \frac{1}{(2 \pi)^{4}} \int_{-\pi}^{+\pi} d \boldsymbol{q} d \boldsymbol{k} \frac{f\left[t_{f \boldsymbol{k}}, \mu_{f}, \beta\right]-f\left[t_{f \boldsymbol{k}+\boldsymbol{q}}, \mu_{f}, \beta\right]}{t_{f \boldsymbol{k}}-t_{f \boldsymbol{k}+\boldsymbol{q}}-\omega+i \epsilon} \\
& =\lim _{\epsilon \rightarrow 0^{+}} \int_{-\infty}^{+\infty} d x \int_{-\infty}^{+\infty} d y \frac{f\left(x, \mu_{f}, \beta\right)-f\left(y, \mu_{f}, \beta\right)}{x-y-\omega+i \epsilon} \rho(x) \rho(y) \\
& =\int_{-\infty}^{+\infty} d \omega^{\prime} \frac{A\left(\omega^{\prime}, \mu_{f}\right)}{\omega^{\prime}-\omega}
\end{aligned}
$$

In the low temperature limit $T \rightarrow 0$ the Fermi distribution becomes $f(x)=1-\Theta(x)$ and we write

$$
\begin{aligned}
A\left(\omega^{\prime}, \mu_{f}\right) & =\chi^{\prime \prime}\left(\omega^{\prime}, \mu_{f}\right) \\
& =\int_{-\infty}^{+\infty} d x\left[\Theta\left(x-\mu_{f}-\omega^{\prime}\right)-\Theta\left(x-\mu_{f}\right)\right] \\
& \times \rho(x) \rho\left(x-\omega^{\prime}\right)
\end{aligned}
$$

that satisfies sum rule $\int_{-\infty}^{+\infty} d \omega A(\omega)=1$. Therefore, we can also calculate the real part

$$
\begin{aligned}
\chi^{\prime}\left(\omega, \mu_{f}\right) & =\mathcal{P} \int_{-\infty}^{+\infty} \frac{d \omega^{\prime}}{\pi} \frac{A\left(\omega^{\prime}, \mu_{f}\right)}{\omega^{\prime}-\omega} \\
& =\frac{2}{\pi} \int_{0}^{+\infty} \frac{\omega^{\prime} \chi^{\prime \prime}\left(\omega^{\prime}, \mu_{f}\right)}{\omega^{\prime 2}-\omega^{2}} d \omega^{\prime} .
\end{aligned}
$$

From these results one finds that $\chi^{\prime}\left(\omega, \mu_{f}\right)$ is an even function of frequency while $\chi^{\prime \prime}\left(\omega, \mu_{f}\right)$ is odd. 


\section{Appendix C: Fermionic number of particles in the effectively interacting system}

In the non-interacting case the number of fermions on the lattice can be calculated as follows

$$
\begin{aligned}
n_{\mathrm{F}} & =\frac{1}{N} \sum_{\boldsymbol{k}} \frac{1}{\exp \left[\beta\left(t_{f \boldsymbol{k}}-\mu_{f}\right)\right]+1} \\
\stackrel{T \rightarrow 0}{=} & \frac{1}{N} \sum_{\boldsymbol{k}} \int_{-\infty}^{+\infty} d \xi \delta\left(\xi-t_{f \boldsymbol{k}}\right)\left[1-\Theta\left(\xi-\mu_{f}\right)\right] \\
& =1-\int_{-\infty}^{+\infty} d \xi \rho(\xi) \Theta\left(\xi-\mu_{f}\right)
\end{aligned}
$$

with $\rho(\xi)$ being the density of states for chosen lattice geometry. Introducing a shift Eq. 62 in the above we are able to obtain the particular value of the chemical potential for fermions with effective interaction induced by the coupling with bosonic species.

\section{Acknowledgments}

We are grateful to N. Teichmann for providing the diagrammatic perturbation theory data used in Table. We thank R. Micnas for fruitful, stimulating discussions that allowed to improve some parts of the paper.
[1] C. Ospelkaus, S. Ospelkaus, K. Sengstock, and K. Bongs, Phys. Rev. Lett. 96, 020401 (2006).

[2] F. Ferlaino, C. D'Errico, G. Roati, M. Zaccanti, M. Inguscio, and G. Modugno, A. Simoni. Phys. Rev. A 73, 040702(R) (2006).

[3] K. Günter, T. Stöferle, H. Moritz, M. Köhl, and T. Esslinger, Phys. Rev. Lett. 96, 180402 (2006).

[4] Th. Best, S. Will, U. Schneider, L. Hackermüller, D. van Oosten, and I. Bloch, D.-S. Lühmann, Phys. Rev. Lett. 102, 030408 (2009).

[5] S. Inouye, M. R. Andrews, J. Stenger, H.-J. Miesner, D. M. Stamper-Kurn, W. Ketterle, Nature 392, 151 (1998).

[6] A. Galindo and P. Pascual, Quantum Mechanics, (Springer-Verlag, 1990).

[7] M. Lewenstein, L. Santos, M. A. Baranov, and H. Fehrmann, Phys. Rev. Lett. 92, 050401 (2004).

[8] A. Albus, F. Illuminati, and J. Eisert, Phys. Rev. A 68, 023606 (2003).

[9] A. Mering and M. Fleischhauer, Phys. Rev. A 81, 011603(R) (2010).

[10] L. Mathey, D. -Wang, W. Hoftstetter, M. D. Lukin and E. Demler, Phys. Rev. Lett. 93, 120404 (2004).

[11] H. P. Büchler and G. Blatter, Phys. Rev. Lett. 91, 130404 (2003).

[12] I. Titvinidze, M. Snoek and W. Hoftstetter, Phys. Rev. Lett. 100, 100401 (2008).

[13] P. P. Orth, D. L. Bergman, and K. Le Hur, Phys. Rev. A 80, 023624 (2009).

[14] M. P. A. Fisher, P. B. Weichman, G. Grinstein, and D. S. Fisher, Phys. Rev. B 40, 546 (1989).

[15] P. Capuzzi, A. Minguzzi, and M. P. Tosi, Phys. Rev. A 68, 033605 (2003).

[16] Z. Akdeniz, M. P. Tosi, Z. Phys. Chem. 217, 927 (2003).

[17] G. Mazzarella, Eur. Phys. J. D 50, 61 (2008).

[18] R. M. Lutchyn, S. Tewari, and S. Das Sarma, Phys. Rev. A 79, 011606(R) (2009).

[19] S. Tewari, R. M. Lutchyn, and S. Das Sarma, Phys. Rev.
B 80, 054511 (2009).

[20] Z. Akdeniz and P. Vignolo, Phys. Lett. A, 373, 2471 (2009).

[21] G. Refael and E. Demler, Phys. Rev. B 77, 144511 (2008).

[22] M. Iskin and J. K. Freericks, Phys. Rev. A 80, 053623 (2009).

[23] S. K. Yip, Phys. Rev. A 64, 023609 (2001).

[24] Jiu-Rong Han, Y. Z. Wang and W. M. Liu, J. Phys. B. 38, 1411 (2005).

[25] K. V. Krutitsky, M. Thorwart, R. Egger and R. Graham, Phys. Rev. A 77, 053609 (2008).

[26] J. Catani, L. De Sarlo, G. Barontini, F. Minardi, and M. Inguscio, Phys. Rev. A 77, 011603(R) (2008).

[27] P. Buosante, S. M. Giampaolo, F. Illuminati, V. Penna and A. Vezzani, Phys. Rev. Lett. 100, 240402 (2008).

[28] T. K. Kopeć, Phys. Rev. B 70, 054518 (2004).

[29] T. P. Polak and T. K. Kopeć, Phys. Rev. B 76, 094503 (2007).

[30] L. Viverit, C. J. Pethick, and H. Smith, Phys. Rev. A 61, 053605 (2000).

[31] G. Giuliani, G. Vignale, Quantum Theory of the Electron Fluid, (Cambridge, 2005).

[32] A. P. Kampf, G. T. Zimanyi, Phys. Rev. B 47, 279 (1993).

[33] M. Abramovitz and I. Stegun, Handbook of Mathematical Functions (Dover, New York, 1970).

[34] N. Teichmann, D. Hinrichs, M. Holthaus, and A. Eckardt, Phys. Rev. B 79, 100503(R) (2009).

[35] B. Capogrosso-Sansone, Ş. G. Söyler, N. Prokof'ev and B. Svistunov, Phys. Rev. A 77, 015602 (2008).

[36] H. P. Büchler and G. Blatter, Phys. Rev. A 69, 063603 (2004).

[37] F. Illuminati and A. Albus, Phys. Rev. Lett. 93, 090406 (2004). 